\title{
Liquid sloshing in a horizontally forced vessel with bottom topography
}

\author{
by M. R. Turner \\ Department of Mathematics, University of Surrey, \\ Guildford, Surrey GU2 7XH, UK
}

- Abstract -

This paper presents a numerical study of the free-surface evolution for inviscid, incompressible, irrotational, horizontally forced sloshing in a twodimensional rectangular vessel with an inhomogeneous bottom topography. The numerical scheme uses a time-dependent conformal mapping to map the physical fluid domain to a rectangle in the computational domain with a time-dependent aspect ratio $Q(t)$, known as the conformal modulus. The advantage of this approach over conventional potential flow solvers is the solution automatically satisfies Laplace's equation for all time, hence only the integration of the two free-surface boundary conditions is required. This makes the scheme computationally fast, and as grid points are required only along the free-surface, high resolution simulations can be performed which allows for simulations for mean fluid depths close to the shallow water water regime. The scheme is robust and can simulate both resonate and non-resonate cases, where in the latter, the large amplitude waves are well predicted.

Results of nonlinear simulations are presented in the case of non-breaking waves for both an asymmetrical 'step' and a symmetric 'hump' bottom topography. The natural free-sloshing mode frequencies are compared with the small topography asymptotic results of Faltinsen and Timokha (2009) (Sloshing, Cambridge University Press (Cambridge)), and are found to be lower than this asymptotic prediction for moderate and large topography magnitudes. For forced periodic oscillations it is shown that the hump profile is the most effective topography for minimising the nonlinear response of the fluid, and hence this topography would reduce the stresses on the vessel walls generated by the fluid. Results also show that varying the width of the step or hump has a less significant effect than varying its magnitude. 


\section{Introduction}

Being able to accurately predict the free-surface motion of a fluid in a vessel is of practical importance in physical applications. The sloshing effects of a fluid in an externally forced vessel may cause detrimental consequences in many engineering applications. For example, the sloshing of liquid fuel in the fuel tanks of spacecraft or rockets can affect their trajectory or, if the sloshing frequency is close to the natural sloshing frequency of the fuel tank itself, then the high dynamic pressures caused by the resonating fluid could damage the walls of the tank. For more information on aerospace applications see the works of Abramson (1966) and Gerrits (2001).

In general, a three-dimensional vessel, such as a ship floating on the ocean, has 6 degrees of freedom. It has 3 linear translations heave, sway and surge and 3 rotational motions, pitch roll and yaw. Understanding the response of the vessel, and the fluid it contains, to each of these 6 different degrees of freedom is vital to fully understand the stability properties of the coupled system (Faltinsen and Timokha, 2009). In the present work we simplify this general situation to consider only 1 linear translation degree of freedom and focus on understanding the free-surface evolution caused by horizontal vessel motion only, such as in Tuned Liquid Dampers (TLDs).

Tuned Liquid Dampers are vessels which contain a fluid which are designed to suppress wind and earthquake oscillations in tall buildings (Kareem et al., 1999). A schematic of a TLD is given in figure 1. The designers of TLDs are responsible for understanding the complicated dynamic coupled motion of the fluid-vessel interaction in order to determine the optimal amount of fluid in the TLD to damp the most severe oscillations. Such an investigation would be costly via experiments alone, hence having an effective numerical scheme which can simulate various forcing frequencies and vessel topologies is beneficial.

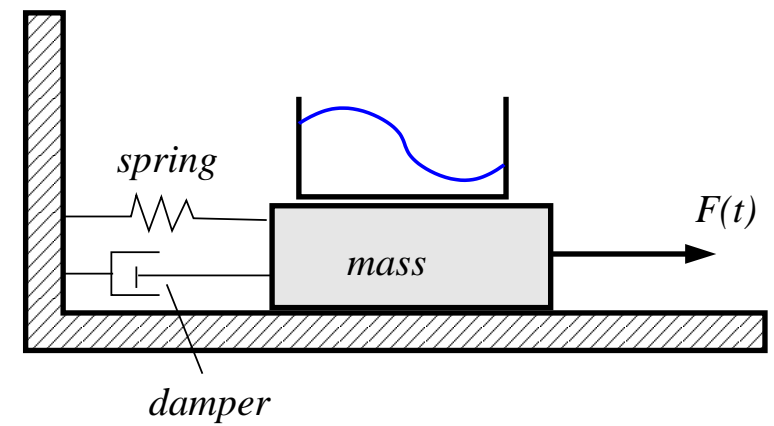

Figure 1: A schematic illustration of a Tuned Liquid Damper (TLD).

Studying the sloshing motion in a stationary or forced vessel either experimentally, theoretically or numerically is very complicated. The works of Moiseyev and Rumyantsev (1968), Ibrahim (2005) and Faltinsen and Timokha (2009), and the references herein, highlight many of the problems observed in this area. The main theoretical and numerical difficulty is accurately calculating the position of the free-surface, which is an unknown of the problem. Previous studies of this problem have tended to use one of two approaches for following the evolution of the free-surface. The first approach uses Lagrangian particle tracking of the numerical nodes on the free-surface with regridding, but the disadvantage of this approach is the surface velocities are difficult to accurately calculate and so the 
surface has to be smoothed. The second approach uses mappings to map the physical domain to a rectangular computational domain with the free-surface now aligned with one edge of the rectangle. This approach requires no smoothing but it cannot easily predict flow features such as wetting and drying of the vessel bottom. However, for most physical applications this is not a major restriction of the method. This mapping approach was successfully implemented by Frandsen (2004) who used a $\sigma$-transformation to map the liquid domain onto a fixed rectangular computational domain (Phillips, 1957) for twodimensional, inviscid, incompressible, irrotational sloshing in a rectangular vessel with a flat bottom. In the computational domain a transformed version of Laplace's equation was solved on a rectangular grid for the velocity potential $\phi$, with the appropriate boundary conditions on the vessel walls and the free-surface. Frandsen (2004) demonstrated that this approach was successful for a flat bottomed rectangular vessel by verifying free-surface results against weakly nonlinear results. However, this $\sigma$-transform approach is limited in two ways: firstly, by solving the transformed version of Laplace's equation directly in the interior of the domain, a two-dimensional numerical grid is required, thus restricting the computational resolution of the method, and secondly, the given $\sigma$-transformation does not extend easily to vessels with inhomogeneous bottom topographies. The numerical approach used in this paper overcomes both these shortfalls and thus is significant to research in this area.

The numerical scheme used in this paper uses a time-dependent conformal mapping to map the physical domain to a rectangular computational domain with time-dependent aspect ratio, $Q(t)$, known as the conformal modulus. As the mapping is conformal the coordinates in the physical domain $x(\mu, \nu, t)+\mathrm{i} y(\mu, \nu, t)$ and the complex potential $\phi(\mu, \nu, t)+\mathrm{i} \psi(\mu, \nu, t)$ are time-dependent holomorphic functions of the computational domain coordinates $(\mu, \nu)$, so they satisfy the Cauchy-Riemann equations

$$
x_{\nu}=-y_{\mu}, \quad x_{\mu}=y_{\nu}, \quad \phi_{\nu}=-\psi_{\mu}, \quad \phi_{\mu}=\psi_{\nu},
$$

and hence they all satisfy Laplace's equation

$$
x_{\mu \mu}+x_{\nu \nu}=0, \quad y_{\mu \mu}+y_{\nu \nu}=0, \quad \phi_{\mu \mu}+\phi_{\nu \nu}=0, \quad \psi_{\mu \mu}+\psi_{\nu \nu}=0
$$

in the computational domain. Here $\psi$ is the corresponding streamfunction to the velocity potential $\phi$. Because of (1.1) and (1.2) we can construct a numerical scheme such that we only calculate the evolution of the two harmonic functions $y$ and $\phi$ on the free-surface, and use integral transforms to relate these functions to the conjugate harmonic functions $x$ and $\psi$ along the free-surface (Turner and Bridges, 2015; Dyachenko et al., 1996, 1999; Choi and Camassa, 1999). When the fluid depth in the vessel is infinite, the integral transforms are just the Hilbert transform (Dyachenko et al., 1996; Papamichael and Stylianopoulos, 2010), but for finite depth fluids the transforms are given by the Hilbert-Garrick transform and depend upon the conformal modulus $Q(t)$ making the transforms time dependent (Turner and Bridges, 2015). The importance of calculating the conformal modulus as part of the numerical scheme is highlighted by Turner and Bridges (2015) who show that fixing the conformal modulus produces results which deviate from results generated using the $\sigma$-transformation numerical scheme of Frandsen (2004) by a larger amount compared to results with a time-dependent conformal modulus. As the mapping in this paper is time-dependent and calculated as part of the solution, it does not have to be stipulated in advance, as in Frandsen (2004), hence we are not restricted to vessels with 
an inhomogeneous bottom topography. In this paper, simulations are presented for a range of intermediate mean fluid depths, in particular the borderline case to deep water and the borderline case to shallow water. For the deep water borderline case, we are able to make direct comparisons with results via the numerical scheme of Frandsen (2004). However, for the borderline case to shallow water we were able to conduct simulations for a smaller mean fluid depth than the Frandsen (2004) code is able to simulate. This is because the current scheme is able to be run with a higher spatial resolution because the harmonic functions $y$ and $\phi$ only need to be solved for along the free-surface and no numerical grid points are required in the interior of the domain. Thus this approach has clear computational advantages and we use it in this paper to investigate the effect of the vessel topography on the fluid free-surface evolution in a periodically forced vessel.

Time-dependent conformal mappings have been used previously to investigate the effect of an inhomogeneous bottom topography on the propagation of periodic water waves (Ruban, 2004, 2005; Viotti et al., 2013). These studies present numerical approaches similar to that presented in this paper, however they do not highlight the time-dependence of the conformal modulus, and its significance on the numerical calculation. It is possible to use non-conformal mappings in the numerical scheme in this paper, with the benefit that then three-dimensional problems can be considered. However in this case the coordinates $(x, y)$ as well as $\phi$ and $\psi$ no longer satisfy Laplace's equation in the computational domain, hence more work is required to solve for the numerical transformation between the conjugate functions. The effect of coupled fluid/vessel sloshing by considering only 1 rotational degree of freedom, roll, using a time-dependent conformal mapping was investigated by Turner et al. (2015b).

The time-dependent conformal mapping technique presented here is most effective for small to moderate amplitude waves, and thus we focus on this regime in this paper. For large amplitude waves and waves with sharp interfaces, the conformal mapping technique is less effective (due to surface grid points being poorly distributed at the sharp crests) and in these cases, methods such as the boundary element method are more effective (e.g. Wilkening and $\mathrm{Yu}(2012))$.

In the area of liquid sloshing, most of the work which examines the effect of bottom topography on the hydrodynamic response, focusses on vessels with submerged baffles. These baffles can be considered as the limit of an infinitely thin hump profile, such as that considered in this paper. Evans and McIver (1987) numerically calculated the natural frequency of sloshing modes in a rectangular tank with a central vertical baffle and found that a submerged baffle made no significant modification to the frequency of these modes. In the present study we shall show that for broader hump topographies the modification of the natural frequencies is more distinct. Akyildiz (2012) performed nonlinear simulations of a vertical baffle topography using a numerical algorithm based on the Volume of Fluid method to reconstruct the free-surface and a finite difference scheme for the fluid equations (Armenio and La Rocca, 1996). As well as comparing free-surface elevations with experiments (Akyildiz and Ünal, 2005; Akyildız and Ünal, 2006), the strength of the vortex shed from the baffle was examined and was found to weaken with increasing baffle height. Other experiments on the submerged baffle topography have found that the damping ratio of the system increases as the top of the baffle approaches the free-surface. A similar conclusion was found by Idir et al., 2009 who showed that a hump topography is more effective at attenuating the energy of a forcing than a flat bottomed TLD. The natural frequencies of sloshing modes in baffled three-dimensional tanks has also been 
examined numerically using boundary integral methods (Firouz-Abadi et al., 2008).

The closest numerical study to that presented in this paper was performed by Choun and Yun (1996) who calculated the natural frequencies in a rectangular tank with a submerged rectangular block, and found that the size and position of the block significantly influences the sloshing natural frequencies. A similar conclusion is drawn by this work, but the numerical scheme in this paper is limited to considering smooth profiles, so we are not able to make direct comparisons at this stage. Other bottom topographies considered by experimentalists include sloping side walls (Gardarsson et al., 2001), but again, our code is not able to replicate this study, because of the wetting and drying of the vessel bottom which occurs as the fluid sloshes back and forth.

Another area of current research which considers water waves propagating over bottom topography is that of coastal wave interaction. This research area models the progression of a train of periodic waves, or a single solitary wave up a coastal beach, investigating the hight increase and steepness of the waves (Grilli et al., 1994, 1997; Grilli, 1998; Grilli and Watts, 1999). In this area, Laplace's equation is solved for in the fluid domain and in the aforementioned works, the solution is formulated as a boundary integral equation. By extending the physical domain, as highlighted in $\S 3$ of this work, the conformal mapping approach presented in this paper could also be implemented, potentially allowing for more general bottom topographies to be considered.

The current paper is laid out as follow. In $\S 2$ the governing nonlinear equations of motion are formulated, while $\S 3$ derives the nonlinear PDEs governing the evolution of the free-surface and documents the solution procedure and the numerical scheme. Results of the numerical simulations for both the step and hump bottom topographies are presented in $\S 4$. Concluding remarks and discussions are given in $\S 5$.

\section{Formulation of governing equations}

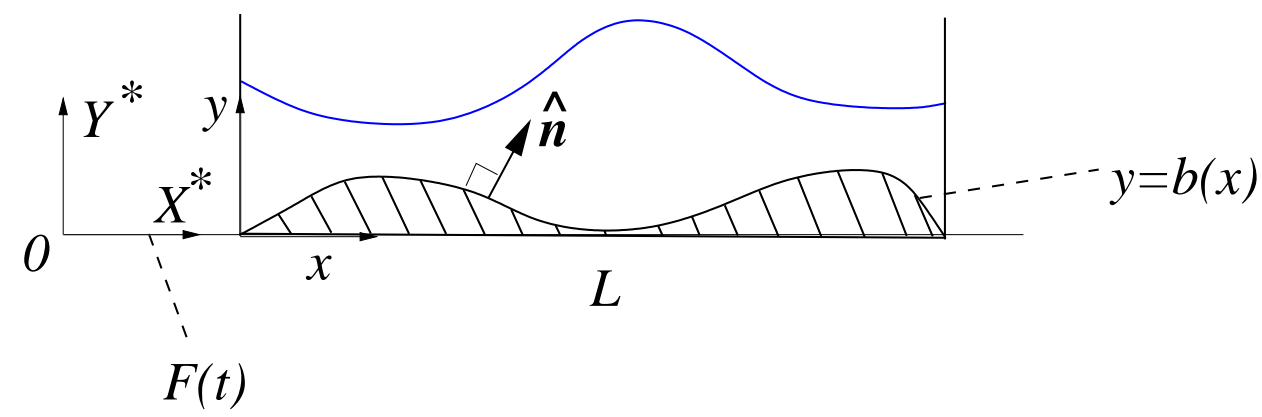

Figure 2: A schematic illustration of forced horizontal sloshing, $F(t)$, in a rectangular vessel with an impermeable bottom at $y=b(x)$.

The governing equations for forced sloshing are given by the Euler equations for the fluid motion, relative to a moving frame. A schematic configuration for this problem is illustrated in figure 2. The tank has length $L$, is of a unit width and has an impermeable bottom given by the function $y=b(x)$, where $(x, y)$ are moving coordinates attached to the vessel, with their origin at the bottom left corner of the vessel. We assume that the fluid motion in the vessel is predominately two-dimensional with the same flow observed 
through each cross-sectional cut in the transverse direction. The moving coordinates are related to the fixed coordinate system $\left(X^{*}, Y^{*}\right)$ via

$$
X^{*}=x+F(t), \quad Y^{*}=y,
$$

where $F(t)$ is a time dependent forcing term to be stipulated. The velocity field relative to the moving frame is denoted by the vector field $\mathbf{u}(\mathbf{x}, t)=(u(x, y, t), v(x, y, t))$. We assume that the base of the vessel remains horizontal throughout the motion, thus the fluid motion is irrotational and the velocity field can be expressed in terms of a velocity potential such that

$$
\phi_{x}=u+\dot{F} \text { and } \phi_{y}=v,
$$

where the velocity potential is relative to the absolute frame (cf. equation (2.1) in Alemi Ardakani et al. (2012)). The governing equations for the fluid motion are then given by

$$
\begin{aligned}
\phi_{x x}+\phi_{y y} & =0, \text { in } 0 \leq x \leq L, b(x) \leq y \leq h(x, t), \\
\phi_{t}+\frac{1}{2}\left(\phi_{x}^{2}+\phi_{y}^{2}\right)-\dot{F} \phi_{x}+g\left(y-h_{0}\right)+\frac{1}{2} \dot{F}^{2} & =B e(t), \text { on } y=h(x, t), \\
h_{t}+\left(\phi_{x}-\dot{F}\right) h_{x} & =\phi_{y}, \text { on } y=h(x, t), \\
\nabla \phi \cdot \widehat{\mathbf{n}} & =(\dot{F}, 0) \cdot \widehat{\mathbf{n}}, \text { on } y=b(x), \\
\phi_{x} & =\dot{F}, \text { on } x=0, L
\end{aligned}
$$

where $B e(t)$ is the Bernoulli function, $h(x, t)$ is the position of the free surface, which is to be determined, and $\widehat{\mathbf{n}}$ is the unit inward normal at the vessel bottom. The derivation of these equations follows an approach similar to that used in Alemi Ardakani et al. (2012).

In the above system of equations the subscripts $x, y$ and $t$ denote partial derivatives and the dots denote full derivatives with respect to $t$. Equations (2.6) and (2.7) are the dynamic and kinematic boundary conditions at the free surface $y=h(x, t)$ while $(2.8)$ and (2.9) represent the no penetration boundary conditions on the bottom and side walls of the vessel respectively.

To solve (2.5)-(2.9) we use a time-dependent conformal mapping and Fourier transforms, and thus we require the solution to be periodic. The symmetric sloshing modes are periodic for $x \in[0, L]$, but the antisymmetric modes are not. Thus we extend the domain to $x \in[0,2 L]$ by forming the even extension of the variables $\phi(x, y, t), h(x, t)$ and the solid bottom $b(x)$, making the solution $2 L$ periodic. Therefore for $x \in[L, 2 L]$ the governing equations are

$$
\begin{aligned}
\phi_{x x}+\phi_{y y} & =0, \text { in } L \leq x \leq 2 L, \widehat{b}(x) \leq y \leq h(x, t)(2.1) \\
\phi_{t}+\frac{1}{2}\left(\phi_{x}^{2}+\phi_{y}^{2}\right)+\dot{F} \phi_{x}+g\left(y-h_{0}\right)+\frac{1}{2} \dot{F}^{2} & =B e(t), \text { on } y=h(x, t), \\
h_{t}+\left(\phi_{x}+\dot{F}\right) h_{x} & =\phi_{y}, \text { on } y=h(x, t), \\
\nabla \phi \cdot \widehat{\mathbf{n}} & =(-\dot{F}, 0) \cdot \widehat{\mathbf{n}}, \text { on } y=\widehat{b}(x), \\
\phi_{x} & =-\dot{F}, \text { on } x=L, 2 L
\end{aligned}
$$

where $\widehat{b}(x)=b(2 L-x)$ is the even extension of the bottom topography. These two problems can be combined into a single problem for the unknown quantities $\phi(x, y, t)$ and $h(x, t)$ by introducing the new velocity potential

$$
\Phi(x, y, t)= \begin{cases}\phi(x, y, t)-x \dot{F}(t), & \text { for } \quad 0 \leq x \leq L \\ \phi(x, y, t)-(2 L-x) \dot{F}(t), & \text { for } \quad L \leq x \leq 2 L\end{cases}
$$


leading to the system of equations

$$
\begin{aligned}
\Phi_{x x}+\Phi_{y y} & =0, \text { in } 0 \leq x \leq 2 L, b(x) \leq y \leq h(x, t), \\
\Phi_{t}+\frac{1}{2}\left(\Phi_{x}^{2}+\Phi_{y}^{2}\right)+g\left(y-h_{0}\right)+x \ddot{F} & =B e, \text { on } y=h(x, t) \text { for } 0 \leq x \leq L, \\
\Phi_{t}+\frac{1}{2}\left(\Phi_{x}^{2}+\Phi_{y}^{2}\right)+g\left(y-h_{0}\right)+(2 L-x) \ddot{F} & =B e, \text { on } y=h(x, t) \text { for } L \leq x \leq 2 L, \\
h_{t}+\Phi_{x} h_{x} & =\Phi_{y}, \text { on } y=h(x, t), \\
\nabla \Phi \cdot \widehat{\mathbf{n}} & =0, \text { on } y=b(x), \\
\Phi_{x} & =0, \text { on } x=0,2 L,
\end{aligned}
$$

where it is assumed that $b(x)$ takes the form of its even extension $\widehat{b}(x)$ for $L \leq x \leq 2 L$.

Equations (2.16)-(2.21) above are purely inviscid, and simulations of these equations are suitable for relatively short time periods, such as those considered in this paper, over which the viscosity of the fluid doesn't have time to act significantly. But, in both long time simulations or sloshing simulations with more viscous liquids, the lack of dissipation considered in the model equations could lead to the simulations incorrectly predicting the evolution of the free surface. This issue can be overcome, if desired, by introducing an artificial dissipation term to the dynamic boundary condition or to both the dynamic and kinematic boundary conditions. For example, one could use the dissipation $\Phi_{t} \mapsto \Phi_{t}+\widetilde{\nu} \Phi$ where $\widetilde{\nu}$ is the artificial dissipation (Baker et al., 1989; Dyachenko et al., 2004) or add alternative additional terms to the free-surface boundary conditions (Tuck, 1974; Wang and Joseph, 2006).

In order to solve the system of equations (2.16)-(2.21) we seek a time-dependent conformal mapping to transform the physical domain $x \in[0,2 L], y \in[b(x), h(x, t)]$ to $\mu \in[0,2 L], \nu \in[-Q(t), 0]$ in the computational domain, where $Q(t)$ is the time dependent conformal modulus of the mapping. This conformal modulus is an additional parameter which needs to be determined as part of the solution procedure. Although this parameter has no physical meaning, we present results for its evolution throughout this paper as its correct calculation is vital for generating correct results. For information on the significance of correctly determining the conformal modulus, see Turner and Bridges (2015). An effective numerical scheme for solving this system of equations, including formulating the governing PDEs for the evolution of the free-surface in parametric form is described in the next section.

\section{Time-dependent conformal mapping}

The strategy we use to solve this problem is to assume that there exists a conformal mapping, $w(x+\mathrm{i} y)$, which maps the physical domain $x \in[0,2 L], y \in[b(x), h(x, t)]$ to the computational domain $\mu \in[0,2 L], \nu \in[-Q(t), 0]$ as shown in figure 3 , where $\mathrm{i}=\sqrt{-1}$. Moreover, it is assumed that: $x=0, L$ and $2 L$ are mapped to $\mu=0, L$ and $2 L$ respectively, $y=b(x)$ and $y=\hat{b}(x)$ are mapped to $\nu=-Q(t)$ and $y=h(x, t)$ is mapped to $\nu=0$. The variables $x+\mathrm{i} y$ and $\Phi+\mathrm{i} \Psi$ are assumed to be holomorphic functions and so satisfy the Cauchy-Riemann equations (1.1) and Laplace's equation (1.2). Here $\Psi$ is the corresponding form of (2.15) for the streamfunction $\psi$.

In the computational domain the coordinates $(x(\mu, \nu, t), y(\mu, \nu, t))$ define a parametric free-surface

$$
(X(\mu, t), Y(\mu, t))=(x(\mu, 0, t), y(\mu, 0, t)) \quad \text { for } \quad \mu \in[0,2 L] .
$$



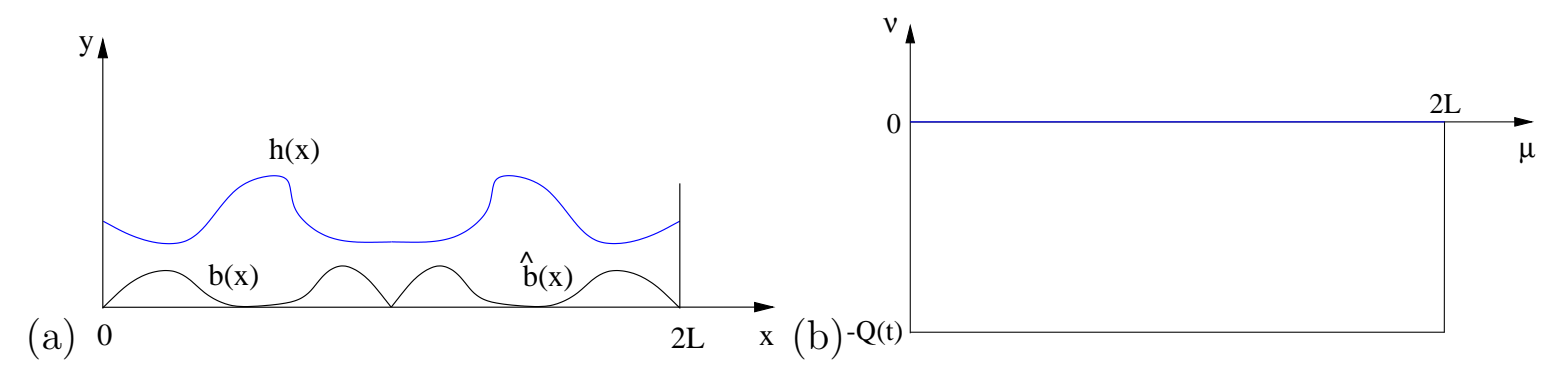

Figure 3: (a) The physical domain and (b) the computational domain.

It is assumed throughout this paper that the free-surface is non-degenerate everywhere, i.e. $J=X_{\mu}^{2}+Y_{\mu}^{2} \neq 0$ for all $t$ and $\mu \in[0,2 L]$.

The governing parametric form of the boundary conditions in the unforced problem are derived in Turner and Bridges (2015), hence following this approach the governing forced boundary conditions become

$$
\begin{aligned}
\frac{1}{J}\left(\Phi_{\mu} x_{\mu}+\Psi_{\mu} y_{\mu}\right) & =0 \text { on } \mu=0,2 L, \\
\Psi_{\mu} & =0 \text { on } \nu=-Q(t), \\
X_{\mu} Y_{t}-Y_{\mu} X_{t} & =-\widetilde{\Psi}_{\mu} \text { on } \nu=0, \\
J \widetilde{\Phi}_{t}-\left(Y_{\mu} Y_{t}+X_{\mu} X_{t}\right) \widetilde{\Phi}_{\mu}+\frac{1}{2}\left(\widetilde{\Phi}_{\mu}^{2}-\widetilde{\Psi}_{\mu}^{2}\right)+g J\left(Y-h_{0}\right)+X \ddot{F} & =0 \text { on } \nu=0, \mu \in[0, L] \\
J \widetilde{\Phi}_{t}-\left(Y_{\mu} Y_{t}+X_{\mu} X_{t}\right) \widetilde{\Phi}_{\mu}+\frac{1}{2}\left(\widetilde{\Phi}_{\mu}^{2}-\widetilde{\Psi}_{\mu}^{2}\right)+g J\left(Y-h_{0}\right)+(2 L-X) \ddot{F} & =0 \text { on } \nu=0, \mu \in[L, 2 L]
\end{aligned}
$$

where

$$
\widetilde{\Phi}(\mu, t)+\mathrm{i} \widetilde{\Psi}(\mu, t)=\Phi(\mu, 0, t)+\mathrm{i} \Psi(\mu, 0, t),
$$

is the complex potential evaluated on the free surface, and the Bernoulli function has been absorbed into the velocity potential.

The free-surface boundary conditions (3.24), (3.25) and (3.26) are implicit equations for the dependent variables $X, Y, \widetilde{\Phi}$ and $\widetilde{\Psi}$, which makes them unsuitable for standard numerical methods. However, following the approach of Turner and Bridges (2015) it is possible to reformulate these implicit equations into explicit equations.

\subsection{Explicit form of the governing free-surface equations}

Note that for any fixed value of $t$, the free-surface $(X(\mu, t), Y(\mu, t))$ is a regular parameterised curve in the plane. Hence any vector in $\mathbb{R}^{2}$ can be uniquely expressed in terms of the vector $\left(X_{\mu}, Y_{\mu}\right)$ and its orthogonal vector $\left(-Y_{\mu}, X_{\mu}\right)$. Therefore

$$
\left(\begin{array}{c}
X_{t} \\
Y_{t}
\end{array}\right)=\frac{\alpha(\mu, t)}{J}\left(\begin{array}{c}
X_{\mu} \\
Y_{\mu}
\end{array}\right)+\frac{\beta(\mu, t)}{J}\left(\begin{array}{c}
-Y_{\mu} \\
X_{\mu}
\end{array}\right)
$$

where $\alpha(\mu, t)$ and $\beta(\mu, t)$ are functions to be determined. Substitution of this expression into (3.24) shows that $\beta=-\widetilde{\Psi}_{\mu}$ but $\alpha$ can be arbitrary. In other words, the tangential velocity of a particle at the surface is arbitrary. Hence a natural explicit form for the kinematic condition is

$$
X_{t}=\frac{\widetilde{\Psi}_{\mu}}{J} Y_{\mu}+\frac{\alpha}{J} X_{\mu}, \quad Y_{t}=-\frac{\widetilde{\Psi}_{\mu}}{J} X_{\mu}+\frac{\alpha}{J} Y_{\mu},
$$


with $\alpha(\mu, t)$ an arbitrary function at this stage. Clearly from these equations we can see that $\alpha=X_{\mu} X_{t}+Y_{\mu} Y_{t}$ which when substituted into (3.25) and (3.26) we find

$$
\begin{array}{ll}
\widetilde{\Phi}_{t}=-g\left(Y-h_{0}\right)-\frac{1}{2 J}\left(\widetilde{\Phi}_{\mu}^{2}-\widetilde{\Psi}_{\mu}^{2}\right)+\frac{\alpha}{J} \widetilde{\Phi}_{\mu}-X \ddot{F} & x \in[0, L], \\
\widetilde{\Phi}_{t}=-g\left(Y-h_{0}\right)-\frac{1}{2 J}\left(\widetilde{\Phi}_{\mu}^{2}-\widetilde{\Psi}_{\mu}^{2}\right)+\frac{\alpha}{J} \widetilde{\Phi}_{\mu}-(2 L-X) \ddot{F} & x \in[L, 2 L] .
\end{array}
$$

Hence the tangential velocity choice $\alpha$ also affects the dynamic free surface boundary condition. In (3.27) and (3.28) the function $\alpha(\mu, t)$ is to be determined, and depends upon the form of the conformal map.

\subsection{Fourier representation of the conformal mapping}

The time-dependent conformal mapping approach used in this paper means that the dependent variables $x, y, \Phi$ and $\Psi$ all automatically satisfy Laplace's equation in the computational domain. Thus the most general form of each of these variables which satisfy the side wall boundary conditions (3.22), have $x=0, L, 2 L$ at $\mu=0, L, 2 L$ and satisfy the Cauchy-Riemann equations (1.1) are

$$
\begin{aligned}
y(\mu, \nu, t) & =h_{0}+C_{0}(t)+\nu+\sum_{n=1}^{\infty}\left(C_{n}(t) \cosh \frac{n \pi \nu}{L}+A_{n}(t) \sinh \frac{n \pi \nu}{L}\right) \cos \frac{n \pi \mu}{L} \\
x(\mu, \nu, t) & =\mu+\sum_{n=1}^{\infty}\left(A_{n}(t) \cosh \frac{n \pi \nu}{L}+C_{n}(t) \sinh \frac{n \pi \nu}{L}\right) \sin \frac{n \pi \mu}{L} \\
\Phi(\mu, \nu, t) & =\beta_{0}(t)+\beta_{01}(t) \nu+\sum_{n=1}^{\infty}\left(\beta_{n}(t) \cosh \frac{n \pi \nu}{L}+\gamma_{n}(t) \sinh \frac{n \pi \nu}{L}\right) \cos \frac{n \pi \mu}{L} \\
\Psi(\mu, \nu, t) & =\gamma_{0}(t)-\beta_{01}(t) \mu+\sum_{n=1}^{\infty}\left(-\gamma_{n}(t) \cosh \frac{n \pi \nu}{L}-\beta_{n}(t) \sinh \frac{n \pi \nu}{L}\right) \sin \frac{n \pi \mu}{L}
\end{aligned}
$$

where $A_{n}, C_{n}, \beta_{n}, \gamma_{n}$ and $\beta_{01}$ are all functions of time to be determined.

Applying the bottom boundary condition (3.23) gives

$$
\left.\Psi_{\mu}\right|_{\nu=-Q(t)}=-\beta_{01}(t)+\sum_{n=1}^{\infty}\left(\beta_{n}(t) \sinh \frac{n \pi Q(t)}{L}-\gamma_{n}(t) \cosh \frac{n \pi Q(t)}{L}\right) \cos \frac{n \pi \mu}{L}=0
$$

which to be true for all $n$ leads to

$$
\beta_{01}(t)=0 \quad \text { and } \quad \gamma_{n}(t)=\beta_{n}(t) \tanh \frac{n \pi Q(t)}{L} .
$$

Thus

$$
\begin{aligned}
& \Phi(\mu, \nu, t)=\beta_{0}(t)+\sum_{n=1}^{\infty} \beta_{n}(t) \frac{\cosh \frac{n \pi}{L}(\nu+Q(t))}{\cosh \frac{n \pi Q(t)}{L}} \cos \frac{n \pi \mu}{L} \\
& \Psi(\mu, \nu, t)=\gamma_{0}(t)-\sum_{n=1}^{\infty} \beta_{n}(t) \frac{\sinh \frac{n \pi}{L}(\nu+Q(t))}{\cosh \frac{n \pi Q(t)}{L}} \sin \frac{n \pi \mu}{L}
\end{aligned}
$$

which agrees with the form of the solutions in Turner and Bridges (2015). The function $\gamma_{0}(t)$ is the value of the streamfunction on the vessel bottom, and as $\Psi$ only appears 
differentiated with respect to $\mu$ in (3.27) and (3.28), we can set $\gamma_{0}(t)=0$ without loss of generality.

The number of unknowns can be further reduced by satisfying the bottom boundary condition for $x(\mu, \nu, t)$ and $y(\mu, \nu, t)$. Therefore on $\nu=-Q(t)$ the dependent variables must satisfy $y=b(x)$ for $x \in[0, L]$ which leads to the nonlinear algebraic equation

$$
\begin{aligned}
& h_{0}-Q(t)+C_{0}(t)+\sum_{n=1}^{\infty}\left(C_{n}(t) \cosh \frac{n \pi Q(t)}{L}-A_{n}(t) \sinh \frac{n \pi Q(t)}{L}\right) \cos \frac{n \pi \mu}{L}= \\
& b\left(\mu+\sum_{n=1}^{\infty}\left(A_{n}(t) \cosh \frac{n \pi Q(t)}{L}-C_{n}(t) \sinh \frac{n \pi Q(t)}{L}\right) \sin \frac{n \pi \mu}{L}\right)
\end{aligned}
$$

which can be written in a more convenient form for numerical computations as

$h_{0}-Q(t)+C_{0}(t)+\sum_{n=1}^{\infty} B_{n}(t) \cos \frac{n \pi \mu}{L}=b\left(\mu+\sum_{n=1}^{\infty}\left(\frac{C_{n}(t)}{\sinh \frac{n \pi Q(t)}{L}}-\frac{B_{n}(t)}{\tanh \frac{n \pi Q(t)}{L}}\right) \sin \frac{n \pi \mu}{L}\right)$,

by using the substitution

$$
A_{n}(t)=\frac{C_{n}(t)}{\tanh \frac{n \pi Q(t)}{L}}-\frac{B_{n}(t)}{\sinh \frac{n \pi Q(t)}{L}} .
$$

Evaluating (3.29)-(3.32) on the free surface $\nu=0$, gives the forms of $X, Y, \widetilde{\Phi}$ and $\widetilde{\Psi}$ which appear in (3.27) and (3.28),

$$
\begin{aligned}
& Y(\mu, t)=h_{0}+C_{0}(t)+\sum_{n=1}^{\infty} C_{n}(t) \cos \frac{n \pi \mu}{L}, \\
& X(\mu, t)=\mu+\sum_{n=1}^{\infty}\left[\frac{C_{n}(t)}{\tanh \frac{n \pi Q(t)}{L}}-\frac{B_{n}(t)}{\sinh \frac{n \pi Q(t)}{L}}\right] \sin \frac{n \pi \mu}{L}, \quad \text { with } B_{n} \text { found via (3.35), } \\
& \widetilde{\Phi}(\mu, t)=\beta_{0}(t)+\sum_{n=1}^{\infty} \beta_{n}(t) \cos \frac{n \pi \mu}{L}, \\
& \widetilde{\Psi}(\mu, t)=\sum_{n=1}^{\infty} \beta_{n}(t) \tanh \frac{n \pi Q(t)}{L} \sin \frac{n \pi \mu}{L},
\end{aligned}
$$

The above equations can be thought of as mappings between the conjugate harmonic functions on the free-surface. Using the notation of $\S 3$ of Turner and Bridges (2015) (Here we just state the relevant results of this paper) we can write these mappings in operator form. The mapping between $\widetilde{\Phi}$ and $\widetilde{\Psi}$ is given by

$$
\widetilde{\Psi}=\mathbf{T}_{q}[\widetilde{\Phi}],
$$

where the operator $\mathbf{T}_{q}[P]$ acting on $P$, which has Fourier series representation

$$
P(\mu)=\frac{a_{0}}{2}+\sum_{n=1}^{\infty}\left(a_{n} \cos \frac{n \pi \mu}{L}+b_{n} \sin \frac{n \pi \mu}{L}\right)
$$

is

$$
\mathbf{T}_{q}[P]=\sum_{n=1}^{\infty} \tanh \frac{n \pi Q}{L}\left(-b_{n} \cos \frac{n \pi \mu}{L}+a_{n} \sin \frac{n \pi \mu}{L}\right)
$$


which is equivalent to that used in Turner and Bridges (2015) for the free sloshing problem.

The mapping between $X$ and $Y$ is more complicated due to the variable bottom and we write this as

$$
X-\mu=\mathbf{U}_{q}^{-1}[Y],
$$

where (3.35) is used to find the $B_{n}$ values in (3.38). In the case of a flat bottomed vessel $(b(x)=0)$ then $B_{n} \equiv 0$ for all $n$, and $\mathbf{U}_{q}^{-1} \equiv \mathbf{T}_{q}^{-1}$, the mapping used by Turner and Bridges (2015).

The form of $\alpha(\mu, t)$, remarkably, can be derived in closed form. As the bottom topography is fixed and so does not have any explicit time dependence, then following the work in Turner and Bridges (2015), the function $z_{t} / z_{\mu}$ has the boundary values

$$
\left.\Im\left(\frac{z_{t}}{z_{\mu}}\right)\right|^{\nu=0}=-\frac{1}{J} \widetilde{\Psi} \text { and }\left.\Im\left(\frac{z_{t}}{z_{\mu}}\right)\right|_{\nu=-Q(t)}=0,
$$

where $z(\mu+\mathrm{i} \nu, t)$. Hence by the Hilbert-Garrick transformation

$$
\alpha=J \bar{\alpha}-J \mathbf{T}_{q}^{-1}\left[\frac{\widetilde{\Psi}}{J}\right]
$$

and we write $\bar{\alpha}$ to mean the mean value of $\alpha$ which can be shown to be equal to zero via the same calculation in Appendix A of Turner and Bridges (2015).

\subsection{Numerical scheme}

The numerical scheme to solve for the free-surface evolution is based on the pseudospectral scheme laid out in Turner and Bridges (2015), and readers are directed there for more information. The free surface is updated by integrating (3.27) and (3.28) using a fourth-order Runge-Kutta scheme with a time step $\Delta t$ and by discretizing $\mu \in[0,2 L]$ using $2 N$ collocation points defined by

$$
\mu_{k}=(k-1) \frac{L}{N}, \quad k=1, \ldots, 2 N .
$$

Derivatives for the variables in (3.27) and (3.28) are evaluated using central finite differences. Discretizing in this way retains $N$ terms in the summations in (3.37)-(3.40).

Equations (3.27) and (3.28) are integrated from some given initial condition,

$$
X_{0}(\mu)+\mathrm{i} Y_{0}(\mu):=X(\mu, 0)+\mathrm{i} Y(\mu, 0), \quad \text { and } \quad \widetilde{\Phi}_{0}(\mu)+\mathrm{i} \widetilde{\Psi}_{0}(\mu):=\widetilde{\Phi}(\mu, 0)+\mathrm{i} \widetilde{\Psi}(\mu, 0),
$$

where the initial values of $X_{0}(\mu)$ and $Y_{0}(\mu)$ at the collocation points come from solving the two coupled nonlinear algebraic expressions

$$
\begin{aligned}
& y=h(x, 0)=H(x) \quad \text { on } \quad \nu=0, \\
& y=b(x) \text { on } \quad \nu=-Q
\end{aligned}
$$

where $H(x)$ is a given free surface elevation. The second of these expressions is equivalent to (3.35), while the first is equivalent to

$$
h_{0}+C_{0}+\sum_{n=1}^{N} C_{n} \cos \frac{n \pi \mu}{L}=H\left(\mu+\sum_{n=1}^{N}\left(\frac{C_{n}(t)}{\tanh \frac{n \pi Q}{L}}-\frac{B_{n}(t)}{\sinh \frac{n \pi Q}{L}}\right) \cos \frac{n \pi \mu}{L}\right) .
$$


This pair of equations are each evaluated at the $N+1$ points $\mu_{k}$ for $k=1, \ldots, N+1$ and the resulting system on $2 N+2$ nonlinear equations are solved via Newton iterations for the $2 N+2$ unknowns $C_{n}, B_{n}$ for $n=1, \ldots, N, C_{0}$ and $Q$.

Time integration of (3.27) and (3.28) from these initial conditions proceeds as follows for each time step. Firstly the function $\alpha(\mu, t)$ is determined by (3.42). Equations (3.27) and (3.28) are now integrated and $X=\mu+\mathbf{U}_{q}^{-1}[Y]$ and $\widetilde{\Psi}=\mathbf{T}_{q}[\widetilde{\Phi}]$ are determined.

After updating all four variables, we update the conformal modulus $Q(t)$. This is also an iterative process as the procedure to determine the forms of $X(\mu, t)$ and $\widetilde{\Psi}(\mu, t)$ depends upon $Q(t)$. The conformal modulus is updated via the $\mu$ independent terms of $(3.35)$ so

$$
Q^{(n)}(t)=h_{0}+C_{0}^{(n)}(t)-\frac{1}{L} \int_{0}^{L} b\left(\mu+\sum_{m=1}^{N}\left[\frac{C_{m}^{(n)}(t)}{\sinh \frac{m \pi Q^{(n)}(t)}{L}}-\frac{B_{m}^{(n)}(t)}{\tanh \frac{m \pi Q^{(n)}(t)}{L}}\right] \sin \frac{m \pi \mu}{L}\right) \mathrm{d} \mu .
$$

Once $Q^{(n)}(t)$ is updated, $X(\mu, t)$ and $\widetilde{\Psi}(\mu, t)$ are updated and this process continues until the relative error in $Q^{(n)}(t)$ is less than some threshold value, which we take to be $10^{-10}$ in $\S 4$. Note, the speed of the iterative processes in the numerical scheme is improved by calculating the Jacobian matrix of the Newton iterations, after the first iteration at the first time step, using the projection method of Broyden (1965).

The results in $\S 4$ are presented with a time step $\Delta t=1 \times 10^{-4}$ and with $N=400$, except for the free-sloshing mode calculations where $N=100$ is used because very small amplitude waves are considered. The nonlinear terms in the governing equations are dealiased, and we apply a filter to $X, Y, \Phi$ and $\Psi$ after each complete time step to suppress growing higher order Fourier modes not removed by the de-aliasing. These additional higher order modes occur due to the highly nonlinear form of (3.27) and (3.28). The reason we are not able to fully de-alias the nonlinear terms, is because the equations contain multiplications by the inverse of a finite Fourier expansion, which itself has an infinite Fourier expansion, and so de-aliasing cannot occur completely. However, testing the numerical scheme for various values of $N$ and different de-aliasing values we found that de-aliasing each quadratic nonlinearity using the $2 N / 3$ approach, and applying the additional filtering, to be sufficient to produce converged results.

All results are found to be independent of larger $N$ and smaller $\Delta t$. This can be seen in figure 4 which shows values of

$$
I_{T}(\Delta t, N)=\int_{0}^{T} \frac{Y(0, t)-h_{0}}{\epsilon} d t
$$

for the nonlinear regime results presented in figures $6(\mathrm{a})(T=15)$ and $9(\mathrm{a})(T=45)$. These results are chosen because they are the largest amplitude results considered in this work, and thus are expected to be the most nonlinear. Therefore these results are expected to be at the top end limit of the numerical scheme. These results show that as $\Delta t$ is reduced and $N$ is increased, the results converge and the resulting free-surface plots are indistinguishable to graphical accuracy (not shown). The slight drop in the value of $I_{T}$ for very small $\Delta t$ values is due to the filtering applied in the numerical scheme to de-alias the nonlinear terms. This drop is tolerable for the results presented in this paper, and as stated above, the results are indistinguishable to graphical accuracy. 

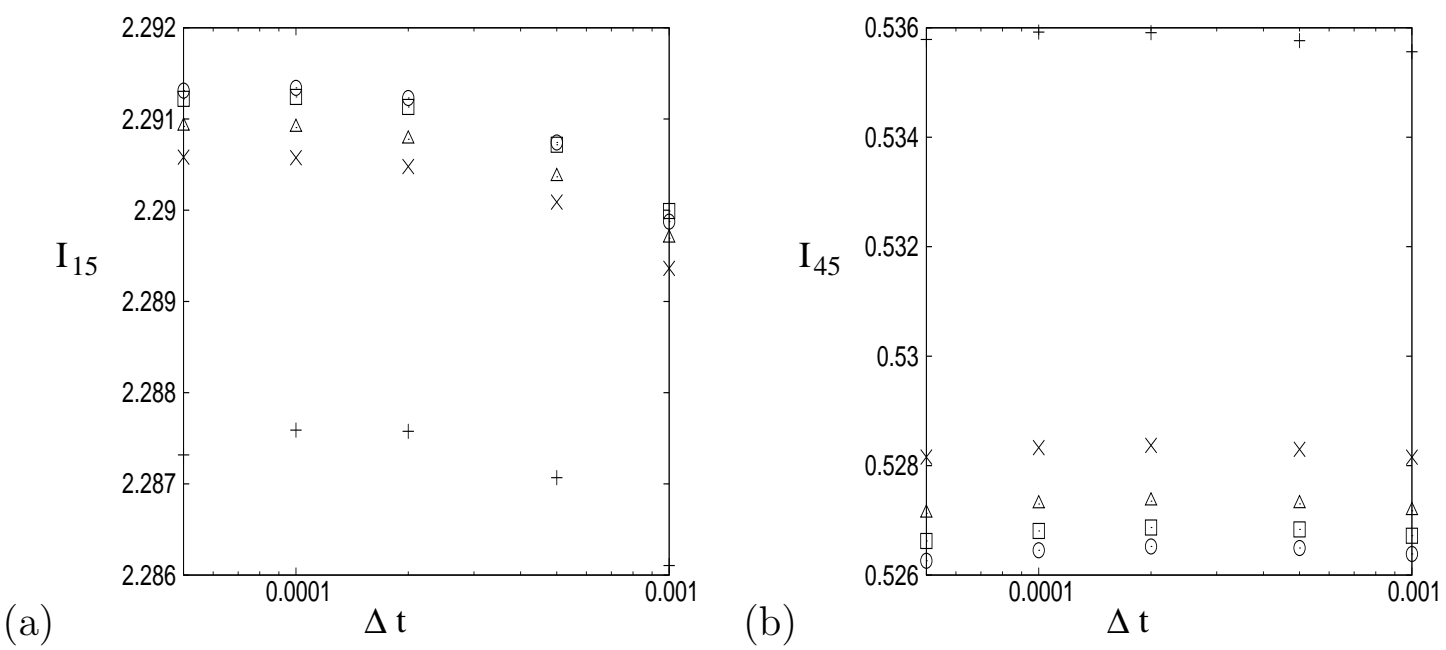

Figure 4: Plot of $I_{T}(\Delta t, N)$ for the nonlinear regime result in (a) figure 6(a) $(T=15)$ for $\delta=0.5$ and (b) figure $9(\mathrm{a})(T=45)$ for $\delta=0.05$. In each panel $+: N=100, \times$ : $N=200, \Delta: N=300, \square: N=400, \odot: N=500$.

All the results presented in $\S 4$ are calculated with a vessel length of $L=0.525 \mathrm{~m}$, taken to coincide with the length of vessel used by Cooker (1994) in his coupled sloshing experiment. Therefore, all dimensional distances $\left(h_{0}, b_{a}, \epsilon, x, y, X, Y\right.$ etc) are measured in $m$, time is measured in $s$ and the frequencies, $\omega$, are measured in $s^{-1}$. Where possible, results are presented using dimensionless variables $\delta=h_{0} / L, \omega t, b_{a} / h_{0}$ and $\bar{Y}(\mu, t) / \epsilon=\left(Y(\mu, t)-h_{0}\right) / \epsilon$, for more generality and thus the results can be scaled up to vessels of an arbitrary length $L$. The frequencies plotted in $\S 4$ are specific for the value of $L=0.525 \mathrm{~m}$ chosen, but these too can be non-dimensionalised, and then scaled up, by considering the non-dimensional frequency $s=\frac{1}{2} \omega L / \sqrt{g h_{0}}$ (Cooker, 1994; Alemi Ardakani et al., 2012).

\section{Results}

Here we present small amplitude (linear regime) and moderate amplitude (nonlinear regime) results for TLD motion with inhomogeneous bottom.

\subsection{Homogeneous topography: $b(x)=0$}

Before considering an inhomogeneous bottom topography, we first consider the case of a flat bottom in order to validate and seek the limitations of the numerical scheme. We also use this section to justify the approach taken for the natural frequency calculation of the free-modes used in $\S 4.2$ and $\S 4.3$.

For the flat bottom rectangular vessel, the natural frequency of the free-sloshing modes $(F(t) \equiv 0)$ are known exactly via

$$
\omega_{n 0}=\left(\frac{g n \pi}{L} \tanh (n \pi \delta)\right)^{1 / 2},
$$


where $\delta=h_{0} / L$ is the dimensionless height of the fluid, but for a general bottom topography no closed form exists. The modes with $n$ odd are antisymmetric sloshing modes and the free-surface has an approximate rotational symmetry about $\left(L / 2, h_{0}\right)$, while the modes with $n$ even are symmetric sloshing modes and the free-surface has an approximate reflectional symmetry about $x=L / 2$. However, we can calculate these natural frequencies from the numerical simulations by initiating the scheme with

$$
H(x)=10^{-6} \sum_{n=1}^{4} \cos \left(\frac{n \pi x}{L}\right),
$$

and $\widetilde{\Phi}_{0}(\mu)=\widetilde{\Psi}_{0}(\mu)=0$. The first 4 sloshing modes are considered, and these are integrated to a large time value, which we take to be $t=1500$. We require a long time series in order to accurately calculate the free-sloshing mode frequencies. Then we create the frequency spectrum $k(\omega)$ for the free-surface elevation at $x=0$ via

$$
k(\omega)=\int_{0}^{1500}\left(Y(0, t)-h_{0}\right) \cos (\omega t) d t
$$

and read off the free sloshing frequencies at the peaks of this function. For example, see figure $5(\mathrm{~b})$ for the case $h_{0}=0.5 \mathrm{~L}$. The frequency results for the first 4 sloshing freemodes are compared to (4.45) in figure 5(a) for the flat bottomed vessel. The agreement is excellent, justifying the use of this approach for the variable bottom case.

(a)

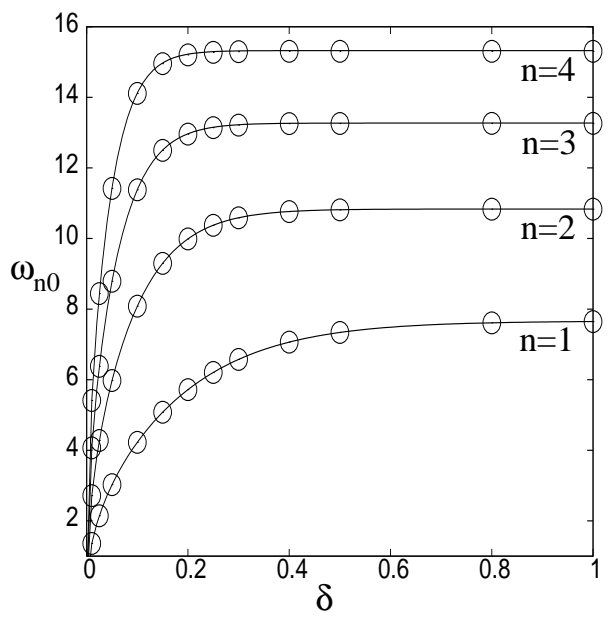

(b)

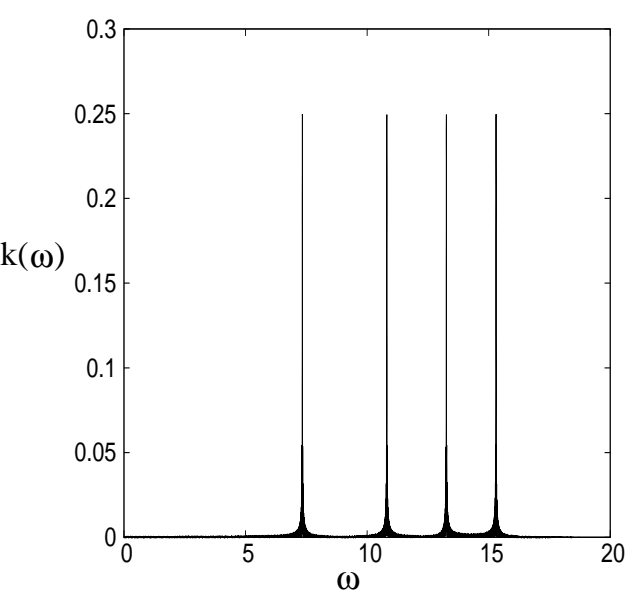

Figure 5: Plot of $(\mathrm{a}) \omega_{n 0}(\delta)$ for $n=1,2,3$ and 4 , where the circles correspond to numerical results, while the solid lines correspond to the analytical result (4.45), and (b) the frequency spectrum, $k(\omega)$, for the case $h_{0}=0.5 \mathrm{~L}$.

For the forced sloshing simulations we use the periodic forcing function

$$
F(t)=\epsilon \cos \left(\omega_{F} t\right)
$$

where $\omega_{F}$ is the forcing frequency and $\epsilon$ is the amplitude. For the initial conditions we consider $H(x)=\widetilde{\Phi}(\mu)=\widetilde{\Psi}(\mu)=0$. This condition generates finite non-zero amplitudes for the asymmetric modes only for symmetric bottoms, and all modes for asymmetric 
bottoms. This is the same form of horizontal forcing considered by Frandsen (2004). Turner and Bridges (2015) show the importance of calculating the time variation of $Q(t)$ for the case $b(x)=0$ and compare their results with the results of Frandsen (2004), thus confirming the validity of the numerical scheme. In figure 6(a) we compare the free-surface elevation $\bar{Y}(\mu, t) / \epsilon=\left(Y(\mu, t)-h_{0}\right) / \epsilon$ directly with those in figure 14(b) of Frandsen (2004), with $h_{0}=0.5 \mathrm{~L}$, and we note the excellent agreement for both the simulations in both the nonlinear (solid line), $\epsilon=0.0078$, and linear (dashed line), $\epsilon=3.0 \times 10^{-4}$, regimes. This excellent agreement is confirmed in figure 6(c) where the result of the current code for $\epsilon=0.0078$ is compared directly with that of Frandsen (2004) (black dots). The nonlinear regime amplitude is chosen to be close to the amplitude where wave breaking first occurs in the simulation. Wave breaking is undesirable as it cannot be resolved by the numerical scheme and thus is beyond the scheme's limitations. In figure 6(b) we plot $Q(t)-h_{0}$ showing the time dependence of the conformal modulus in the numerical calculation, thus showing its significance. Here we also observe the result from Turner and Bridges (2015) who show that the magnitude of $Q(t)-h_{0}$ is directly proportional to the amplitude of the sloshing waves (which in turn are directly proportional to the forcing amplitude). Hence when we are in the nonlinear amplitude regime, the nonlinear effects which occur in the fluid will also be reflected as nonlinear effects in $Q(t)$.

The free-surface profiles for the nonlinear regime result in figure 7(b) show that the amplification of higher order modes in the solution, breaks the rotational symmetry of the profiles when compared to the linear regime profiles in figure 7(a), and gives a steeper 'wave front' appearance to the nonlinear regime profiles.

In figure 8 we plot the interior fluid velocities $u(x, y)=\partial \Phi / \partial x$ and $v(x, y)=\partial \Phi / \partial y$ in the interior of the vessel for two time values. To numerically calculate these velocites, the partial derivatives with respect to $x$ and $y$ are written in terms of derivatives with respect to $\mu$ and $\nu$ via chain rule and (1.1) (Bridges and Donaldson, 2011)

$$
\frac{\partial \Phi}{\partial x}=\frac{1}{J}\left(\frac{\partial \Phi}{\partial \mu} \frac{\partial x}{\partial \mu}+\frac{\partial \Psi}{\partial \mu} \frac{\partial y}{\partial \mu}\right) \text { and } \frac{\partial \Phi}{\partial y}=\frac{1}{J}\left(\frac{\partial \Phi}{\partial \mu} \frac{\partial y}{\partial \mu}-\frac{\partial \Psi}{\partial \mu} \frac{\partial x}{\partial \mu}\right)
$$

and $y, x, \Phi$ and $\Psi$ are evaluated on an $(N+1) \times(N+1)$ grid in the interior of the computational domain via (3.29)-(3.32) respectively. The resulting derivatives of these functions with respect to $\mu$ are then calculated via central finite differences, making then accurate to $O\left(N^{-2}\right)$. The resulting plots show that the largest horizontal velocities occur at points on the free-surface where the steep wave fronts tend to occur.

The simulation above with $h_{0}=0.5 \mathrm{~L}$ is an intermediate mean fluid depth on the deep-fluid border. However, one benefit of this numerical scheme, which only solves the governing equations along the free-surface, is we can consider shallower water levels than can be considered by the Frandsen (2004) scheme. The reason for this is because the Frandsen (2004) scheme could not converge for $h_{0}=0.05 \mathrm{~L}$ for the grid resolutions considered. Higher resolution grids were required, and due to the need for interior grid points in this scheme, the computations soon became unwieldy. In figures 9-11 we present results for the mean fluid depth $h_{0}=0.05 \mathrm{~L}$ which is on the border between an intermediate depth fluid and a shallow fluid. Numerically this fluid depth is more challenging and the numerical scheme fails to converge at fluid depths much smaller than this. At this mean fluid depth, wave breaking occurs more readily, hence the maximum forcing amplitude considered is less than for the $h_{0}=0.5 \mathrm{~L}$ case, but is again chosen to be close to the value where wave breaking occurs. The results in figures 9-11 are qualitatively similar 
(a)

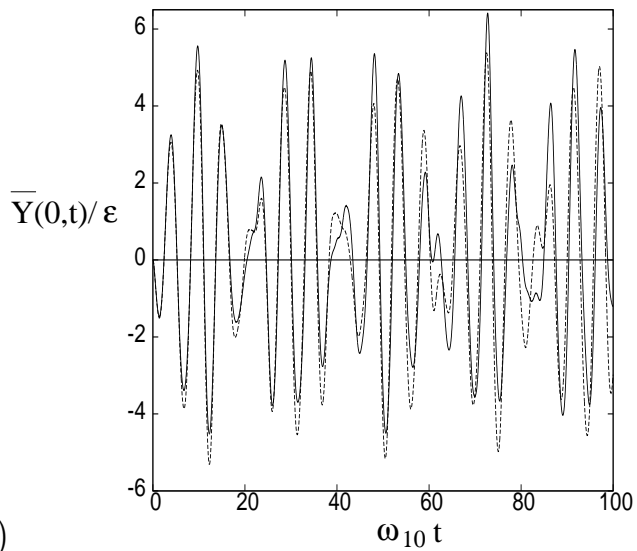

(b)

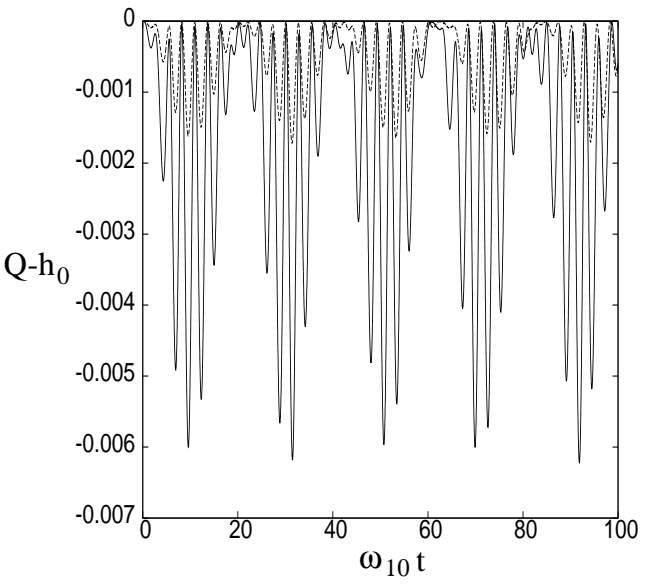

(c)

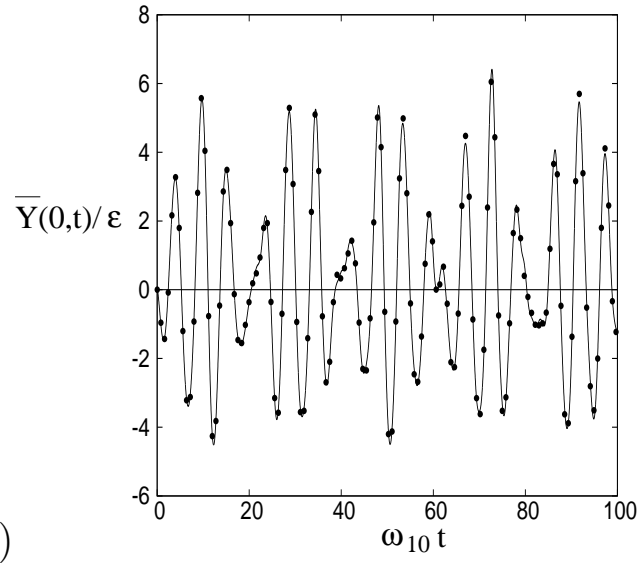

Figure 6: Plot of (a) $\bar{Y}(0, t) / \epsilon$ and (b) $Q(t)-h_{0}$ for the case when $h_{0}=0.5 L, \omega_{F} / \omega_{10}=$ 1.3. In each panel the dashed line corresponds to the linear regime result, $\epsilon=3.0 \times 10^{-4}$, and the solid line is the nonlinear regime result, $\epsilon=0.0078$. In panel (b) the magnitude of the linear regime result is increased by a factor of 100 for a visual comparison. Panel (c) replots the nonlinear regime result of panel (a) and the dots correspond to results using the code of Frandsen (2004).

to the deeper water results, except with higher frequency fluid oscillations, and smaller magnitude peaks in $\bar{Y}(0, t) / \epsilon$. The free-surface profiles in figure 10 are also qualitatively similar, with the linear regime results in panel (a) giving a sinusoidal appearance, and the nonlinear regime results in panel (b) having the steeper wave front appearance during the sloshing motion.

The interior velocity plots in figure 11 show that in this shallower fluid the horizontal velocity is approaching a depth-averaged state where $u(x, y) \approx u(x)$. This confirms that this fluid depth is close to the shallow water limit. In the shallow water limit we have to take care when comparing our inviscid results to experimental results because in this limit the viscous effects on the vessel bottom, neglected by this scheme, become significant and lead to the attenuation of of the waves (Putnam and Johson, 1949). However, viscous effects are not considered in this study.

Finally, to examine the limitation of the numerical scheme with respect to calculating large amplitude waves, we consider the resonance case when $\omega_{F}=\omega_{10}$, with $h_{0}=0.5 \mathrm{~L}$ and $\epsilon=0.0026$ in figure 12. Here again the dots represent simulations of the Frandsen 
(a)

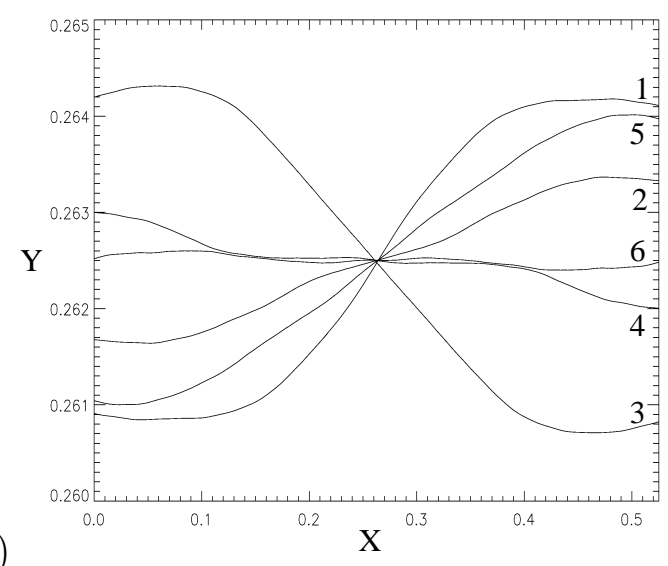

(b)

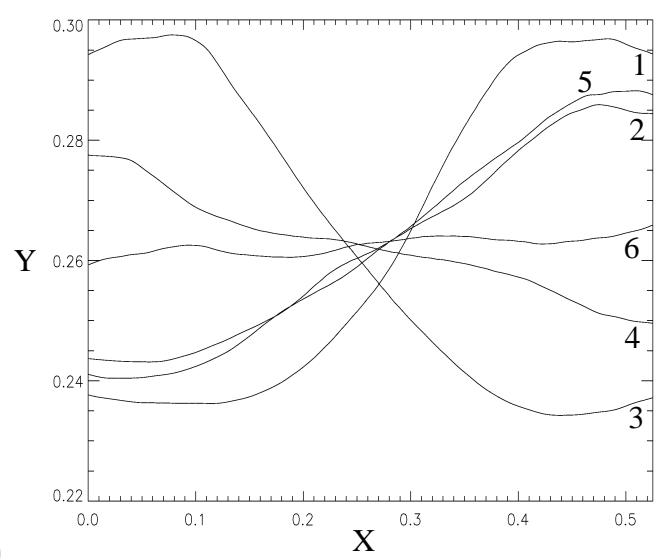

Figure 7: Plot of $Y(X, t)$ for the case when $h_{0}=0.5 L, \omega_{F} / \omega_{10}=1.3$ and (a) $\epsilon=$ $3.0 \times 10^{-4}$ and (b) $\epsilon=0.0078$. In each panel the results for times $t=4.2,4.4,4.6,4.8,5.0$ and $5.2\left(\omega_{10} t=30.8,32.3,33.8,35.2,36.7\right.$ and 38.2) are numbered 1-6 respectively.
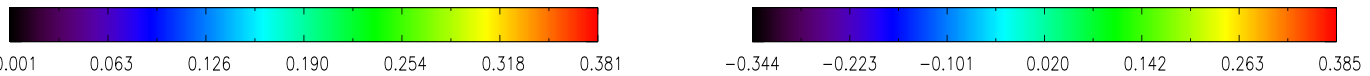

(a)

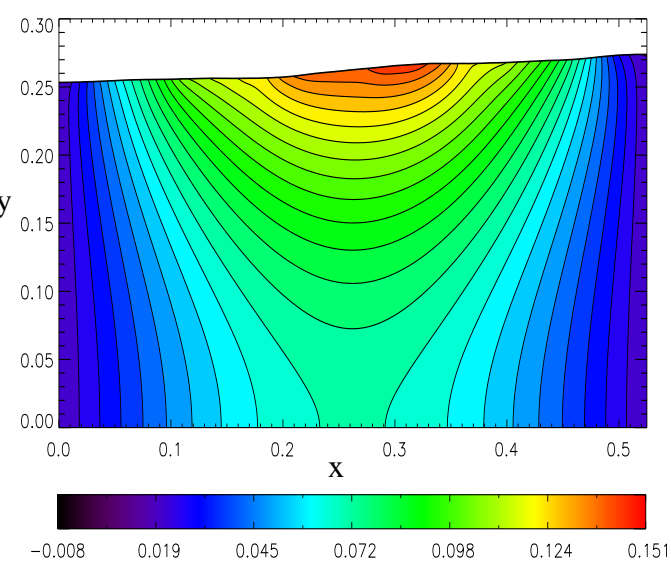

(b)
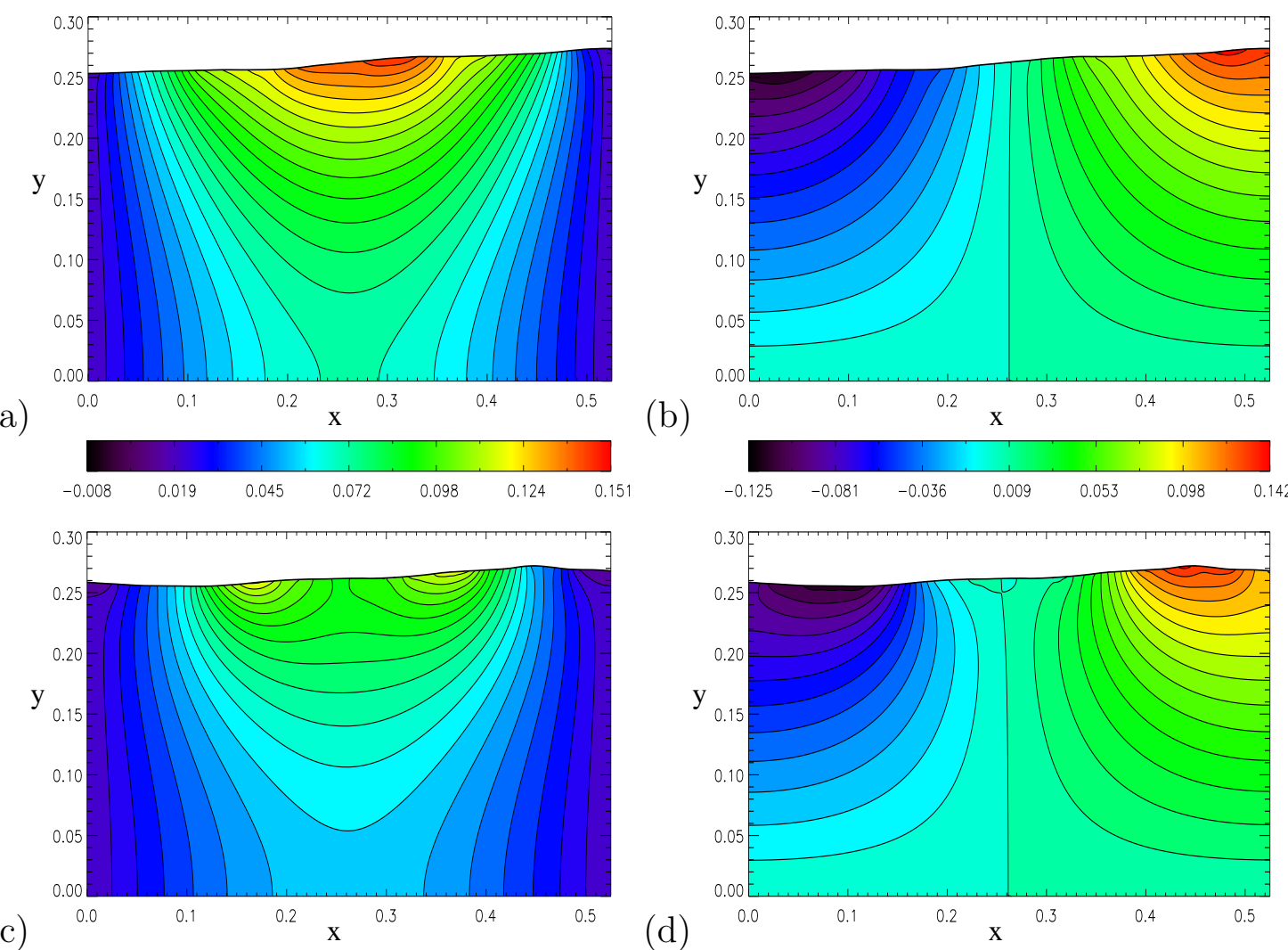

(d)

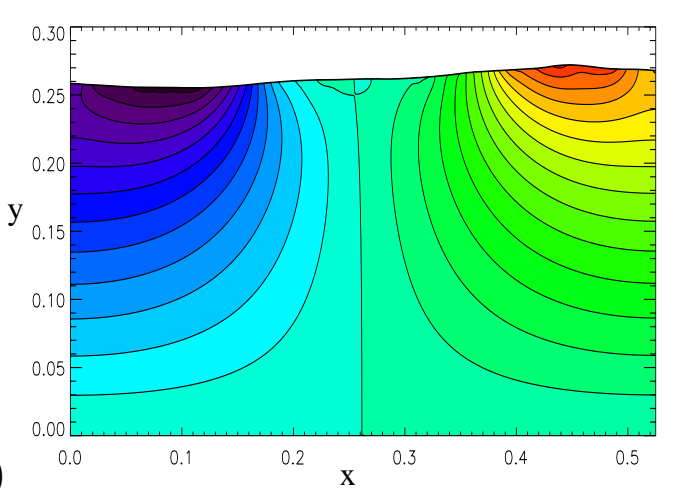

Figure 8: Plot of the contours for (a) $u(x, y)$ and (b) $v(x, y)$ at $t=6.75\left(\omega_{10} t=49.5\right)$ and (c) $u(x, y)$ and (d) $v(x, y)$ at $t=13.5\left(\omega_{10} t=99.1\right)$ for $h_{0}=0.5 L, \omega_{F} / \omega_{10}=1.3$ and $\epsilon=0.0078$. 
(a)

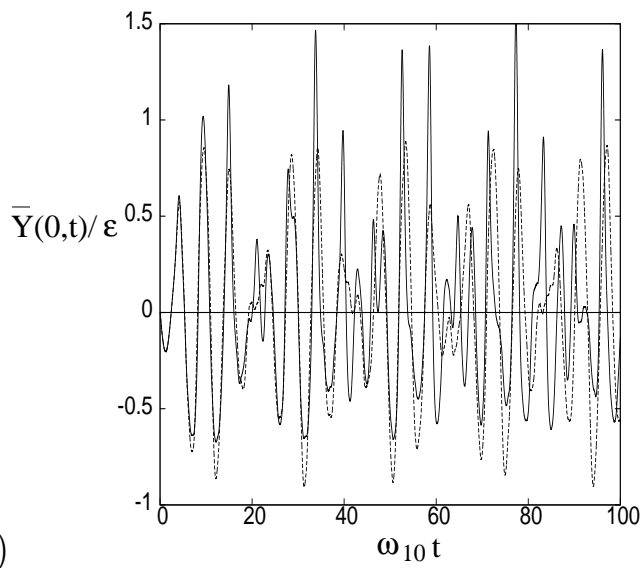

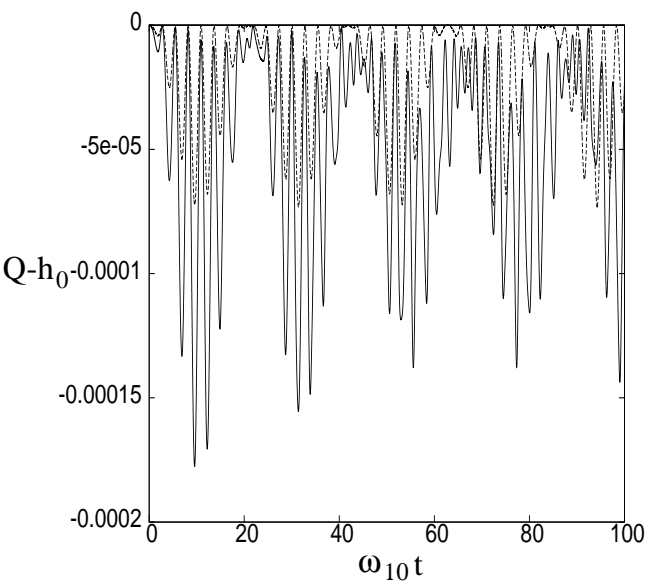

Figure 9: Plot of (a) $\bar{Y}(0, t) / \epsilon$ and (b) $Q(t)-h_{0}$ for the case when $h_{0}=0.05 L, \omega_{F} / \omega_{10}=$ 1.3. In each panel the dashed line corresponds to the linear regime result, $\epsilon=6.3 \times 10^{-5}$, and the solid line is the nonlinear regime result, $\epsilon=0.0032$. In panel (b) the magnitude of the linear regime result is increased by a factor of 1000 for a visual comparison.

(a)

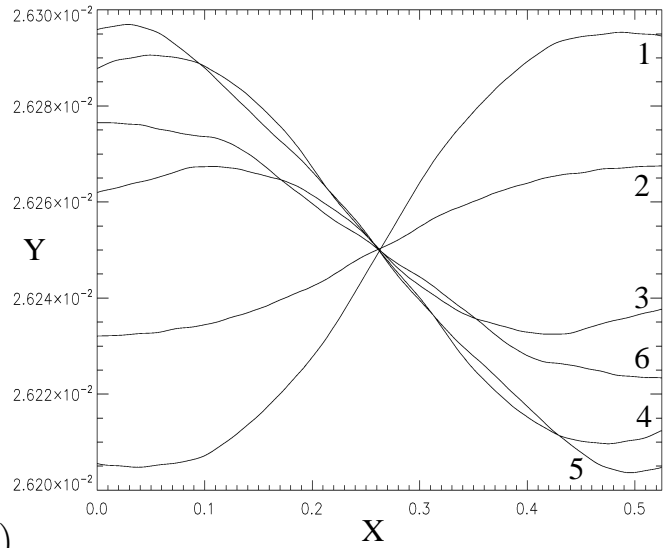

Figure 10: Plot of $Y(X, t)$ for the case when $h_{0}=0.05 L, \omega_{F} / \omega_{10}=1.3$ and (a) $\epsilon=$ $6.3 \times 10^{-5}$ and $(\mathrm{b}) \epsilon=0.0032$. In each panel the results for times $t=4.2,4.4,4.6,4.8,5.0$ and $5.2\left(\omega_{10} t=12.7,13.3,13.9,14.5,15.1\right.$ and 15.7 $)$ are numbered 1-6 respectively.

(2004) numerical scheme. We see that in this case the current scheme does not integrate for as long as the Frandsen scheme, but still manages to calculate wave amplitudes 60 times larger than the forcing amplitude. Figure 12(b) shows that the current scheme breaks down due to not being able to iteratively calculate the conformal modulus due to the rapid, large amplitude, oscillations in $Q(t)$. Further progress to larger time values can be achieved by reducing $\Delta t$ so that the initial guess for $Q(t)$ from the previous time-step is more accurate, but the time-step size soon becomes too small to feasibly run the simulation in a reasonable time. This limitation of the scheme however, is not too limiting, as the main interest in this paper is in non-resonant cases.

With the numerical scheme now justified, and its limitations known, we next investigate any qualitative or quantitative differences to these sloshing results for inhomogeneous 

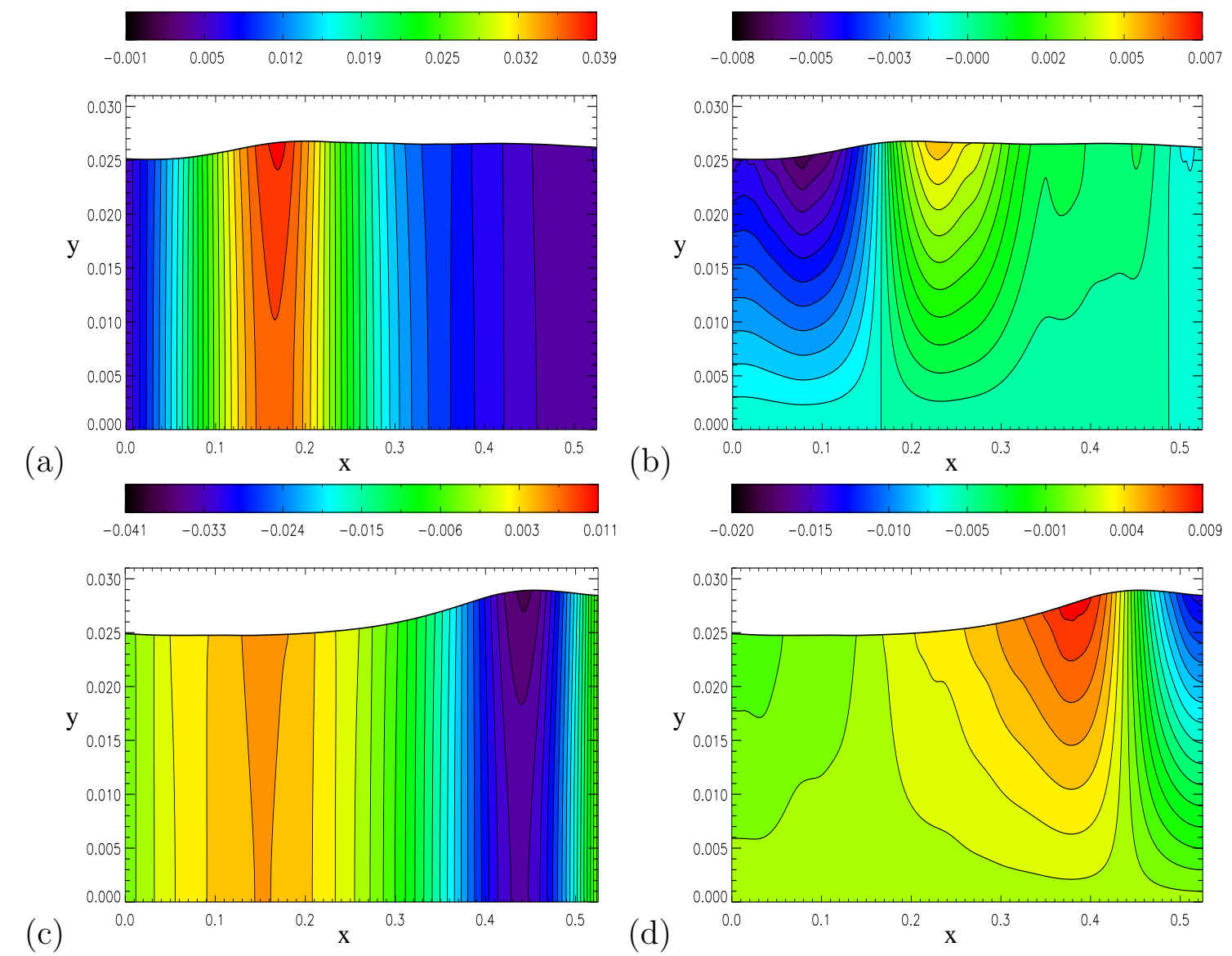

Figure 11: Plot of the contours for (a) $u(x, y)$ and (b) $v(x, y)$ at $t=13.5\left(\omega_{10} t=40.8\right)$ and (c) $u(x, y)$ and (d) $v(x, y)$ at $t=24.75\left(\omega_{10} t=74.8\right)$ for $h_{0}=0.05 L, \omega_{F} / \omega_{10}=1.3$ and $\epsilon=0.0032$.

(a)

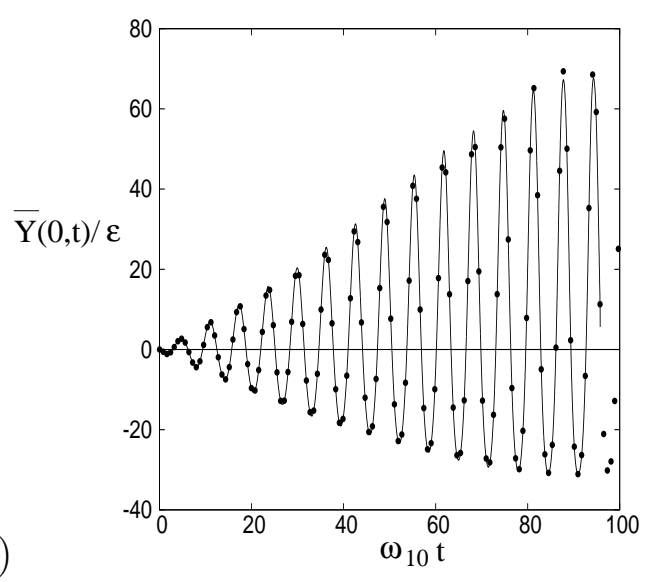

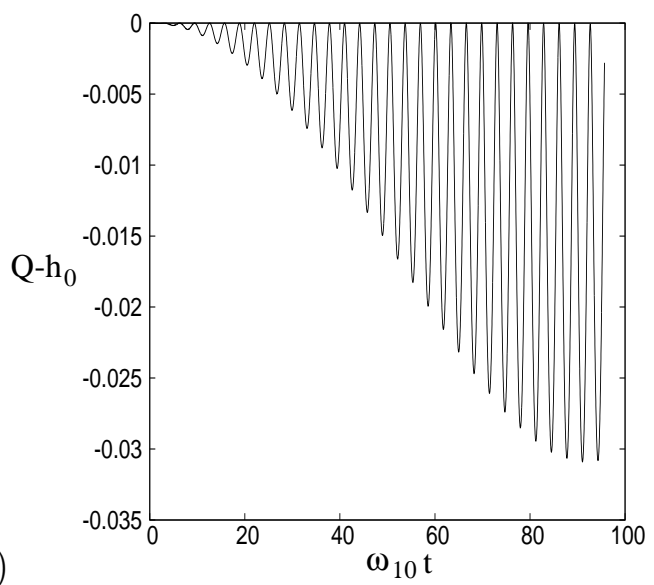

Figure 12: Plot of (a) $\bar{Y}(0, t) / \epsilon$ and (b) $Q(t)-h_{0}$ for the resonance case when $h_{0}=0.5 L$, $\omega_{F} / \omega_{10}=1$ and $\epsilon=0.0026$ (nonlinear regime). In panel (a) the black dots correspond to the results of the code of Frandsen (2004). 
bottom topographies in the following sections.

\subsection{Step topography: $b(x)=\frac{1}{2} b_{a}\left(1-\cos \frac{\pi x}{L}\right)$}

In this section we consider the sloshing motion in a TLD with a step-bottom topography given by

$$
b(x)=\frac{1}{2} b_{a}\left(1-\cos \frac{\pi x}{L}\right) \quad \text { for } \quad 0<x<L,
$$

where $b_{a}$ is a measure of the topography magnitude. An example of this topography is given by curve 2 in figure 13. Here the bottom is asymmetric for $b_{a} \neq 0$ thus we expect

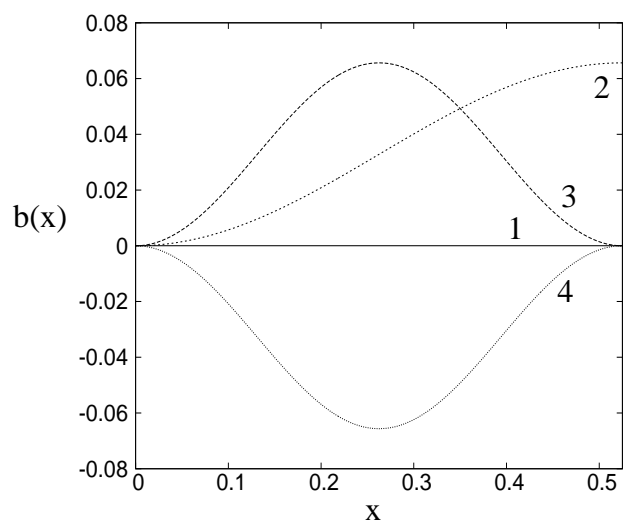

Figure 13: Plot of $b(x)$ for the four topography types considered in $\S 4.1$ (curve 1), $\S 4.2$ (curve 2) and $\S 4.3$ (curves 3 and 4 ) for $b_{a}=\frac{1}{4} h_{0}=\frac{1}{4} \delta L$ and $\delta=\frac{1}{2}$.

different free-surface behaviours at either end of the vessel. Also, as the symmetry of the vessel has been broken, we expect the distinction between symmetric and antisymmetric free sloshing modes not to apply in this case, and in this section we describe them just as 'sloshing modes'. Before investigating the nonlinear fluid effects of the forced TLD, we first investigate how the natural frequencies of the first 4 sloshing modes are modified by increasing the ratio of the step height to the mean fluid depth, $b_{a} / h_{0}$. These results are displayed in figure 14 for (a) $\delta=0.05$, (b) $\delta=0.15$ and (c) $\delta=0.5$. The results of the numerical simulations, given by the symbols, show that as the magnitude of the bottom topography is increased the natural frequency of each mode reduces. This is in agreement with the asymptotic result for $b_{a} / h_{0} \ll 1$ given by equation (4.90) of Faltinsen and Timokha (2009). This states

$$
\omega_{n}^{2}=\omega_{n 0}^{2}\left(1-\frac{\left.\left.\int_{0}^{L} \frac{\partial \phi_{n}}{\partial n}\right|_{y=b(x)} \phi_{n}\right|_{y=b(x)} d x}{\frac{n \pi}{L} \tanh \frac{n \pi h_{0}}{L} \int_{0}^{L}\left(\left.\phi_{n}\right|_{y=h_{0}}\right)^{2} d x}\right),
$$

where

$$
\phi_{n}=\frac{\cosh \frac{n \pi y}{L}}{\cosh \frac{n \pi h_{0}}{L}} \cos \frac{n \pi x}{L},
$$

is the modal form for the free modes for a homogeneous bottomed vessel and $\omega_{n 0}$ is the frequency of the $n^{\text {th }}$ mode for the homogeneous bottomed vessel given in (4.45). 

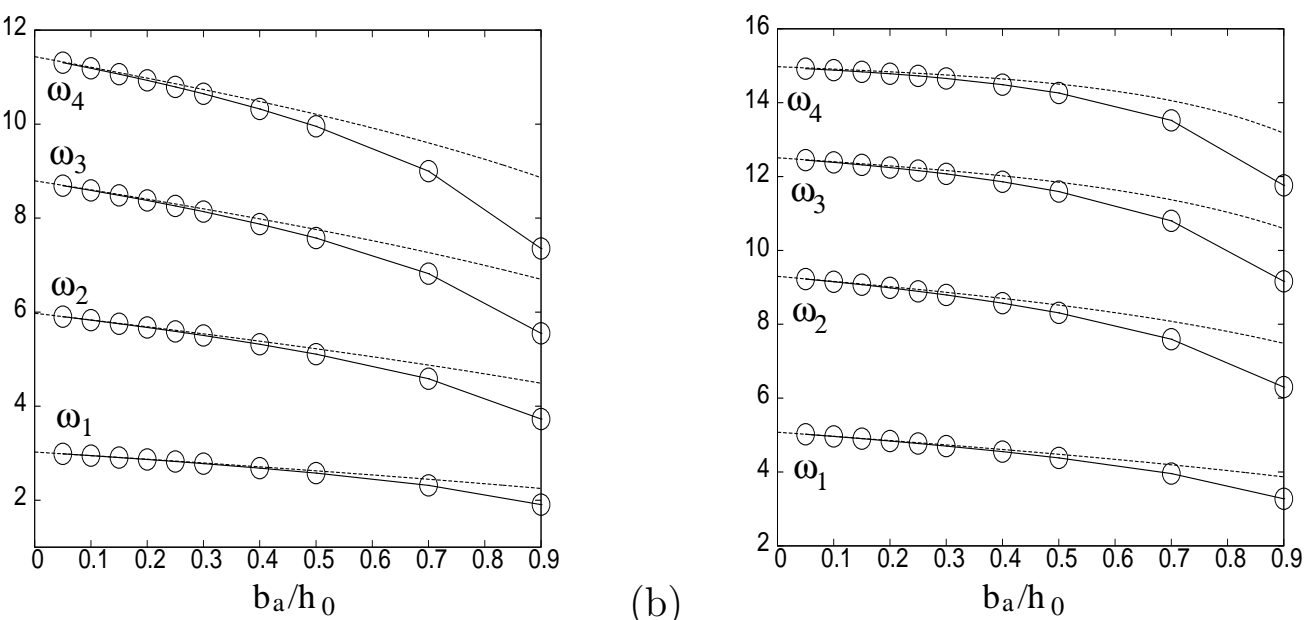

(a)

(b)

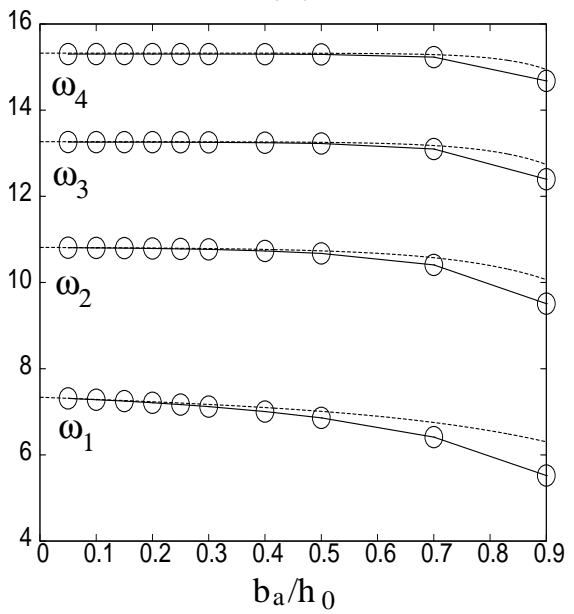

(c)

Figure 14: Plot of the natural frequencies $\omega_{n}\left(b_{a} / h_{0}\right)$ for (a) $\delta=0.05$, (b) $\delta=0.15$ and (c) $\delta=0.5$. In each panel the circles represent the results of the numerical simulations and the dashed lines give the asymptotic result (4.49) for the first 4 modes.

We note that agreement between the numerical and asymptotic results is very good for $b_{a} / h_{0} \lesssim 0.3$ and the asymptotic result over estimates the frequency for larger magnitude steps. The implications of this reduction in the natural frequency of the free-modes is discussed below.

In figure 15 we display both simulations in the linear and nonlinear regimes for the case $h_{0}=0.5 L, \omega_{F} / \omega_{10}=1.3$ with $b_{a} / h_{0}=0,0.25$ and 0.5 . As the bottom topography is asymmetric in this section we display results for the amplitude of the free surface at both ends of the vessel for comparison. Panel (a) shows that at the left-hand end of the vessel (deeper fluid region) the magnitude of the surface elevation decreases as the magnitude of the bottom topography increases, while at the right-hand end of the vessel (shallow fluid region) the magnitude of the free-surface elevation is similar for each value of $b_{a} / h_{0}$. This can more clearly be seen in figure $15(\mathrm{~d})$ where the $b_{a} / h_{0}=0$ result is plotted together with the $b_{a} / h_{0}=0.5$ result. The reason for this different qualitative behaviour is because, at $x=0$ the force on the fluid is directed perpendicular to the wall for $0 \leq y \leq h$, however at $x=L$ the force on the fluid is only directed perpendicular to the wall for $b_{a} \leq y \leq h$, and for $0 \leq y \leq b_{a}$ the force is directed normal to the vessel base, 
(a)

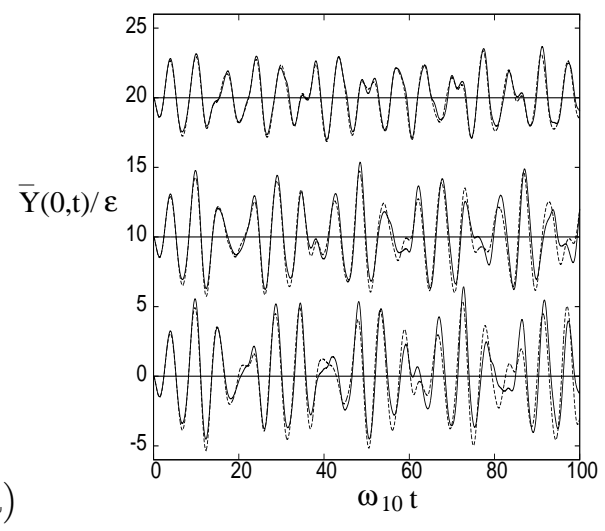

(c)

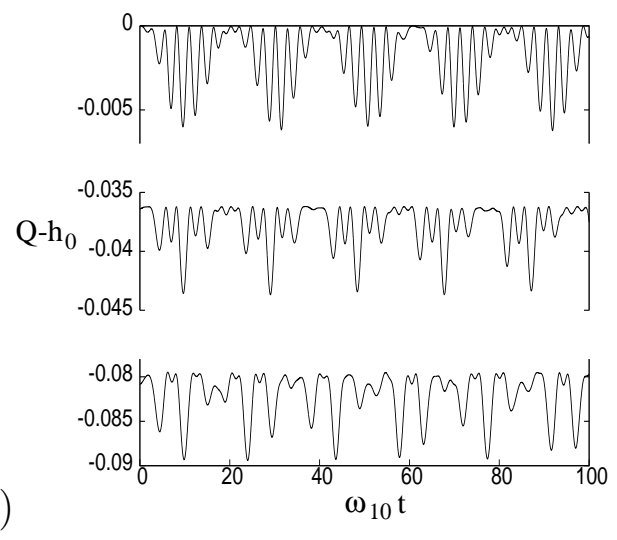

(b)

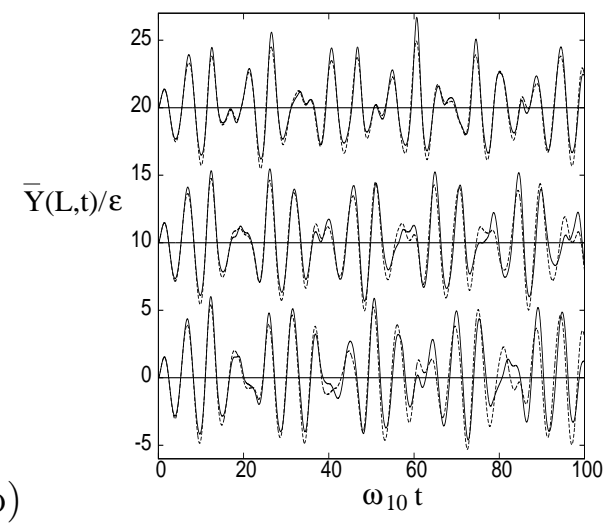

(d)

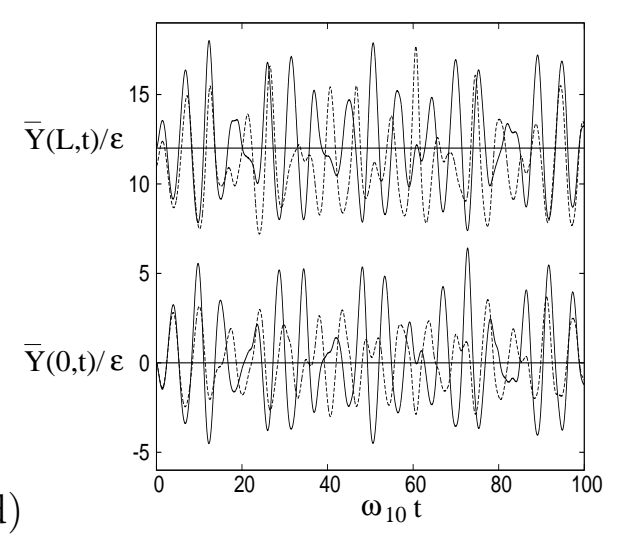

(e)

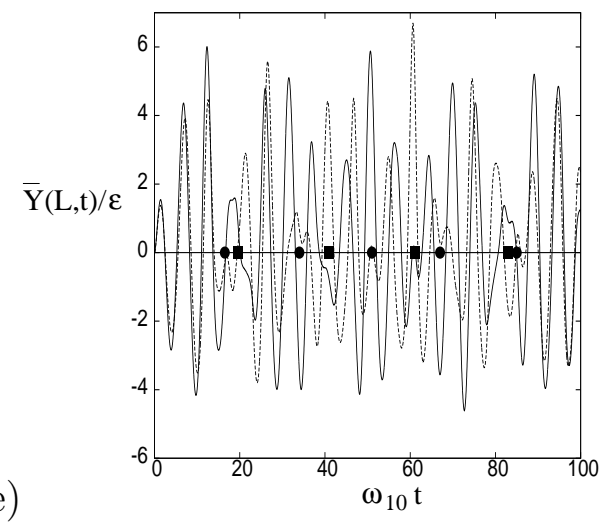

Figure 15: Plot of (a) $\bar{Y}(0, t) / \epsilon$ and (b) $\bar{Y}(L, t) / \epsilon$, when $h_{0}=0.5 L, \omega_{F} / \omega_{10}=1.3$ and $b_{a} / h_{0}=0,0.25$ and 0.5 from bottom to top. In each panel the dashed line corresponds to the linear regime result, $\epsilon=3.9 \times 10^{-4}$, and the solid line is the nonlinear regime result, $\epsilon=0.0078$. Panel (c) plots $Q(t)-h_{0}$ for the nonlinear regime results in panels (a) and (b) with $b_{a} / h_{0}=0,0.25$ and 0.5 from top to bottom. (Note the different $y$-axis scales). Panel (d) plots $\bar{Y}(0, t) / \epsilon$ on the bottom and $\bar{Y}(L, t) / \epsilon$ on the top for $b_{a} / h_{0}=0$ (solid lines) and $b_{a} / h_{0}=0.5$ (dashed lines) in order to show the change in wave amplitudes. Panel (e) plots $\bar{Y}(L, t) / \epsilon$ for $b_{a} / h_{0}=0$ (solid line) and 0.5 (dashed line) on the same axis highlighting the drift in time of when the vessel and the fluid motions are out of phase. These regions occur approximately at the squares for $b_{a} / h_{0}=0$ and at the circles for $b_{a} / h_{0}=0.5$. 
which also contains a component in the vertical direction. Hence at $x=L$ the horizontal force on the fluid by the vessel is less than at $x=0$. This means that as the vessel moves from left to right ( $x$ increasing) a larger amplitude sloshing wave will be formed, giving a larger surface elevation at $x=L$, while when it moves from right to left ( $x$ decreasing) a smaller amplitude wave is formed giving a smaller surface elevation at $x=0$.

In panels (a) and (b) it is also clear that as the magnitude of the bottom topography is increased the magnitude of the nonlinear effects in the fluid are reduced. Thus to see nonlinear fluid effects with a large step topography requires a larger forcing amplitude. In figure 15(b) we also observe that because the frequency of the free-modes is reduced as $b_{a}$ increases (see figure 14 for natural frequencies of the free-modes), the period of time between points where the vessel and the fluid motion are out of phase with one another (which corresponds to regions of smaller surface elevation in between the larger periodic oscillations) is reduced. This can more clearly be seen in figure $15(\mathrm{e})$, where the squares and circles represent the approximate middle point of this region for $b_{a} / h_{0}=0$ and $b_{a} / h_{0}=0.5$ respectively. Thus these simulations suggest that including a step topography in the vessel is an effective way to remove moderate amplitude sloshing modes and hence high stresses on the vessel walls.

The evolution of $Q(t)-h_{0}$ for the three nonlinear regime simulations is given in figure 15(c), and we note that as $b_{a}$ increases, the magnitude of the oscillations in $Q(t)$ also increases, meaning that the correct calculation of $Q(t)$ on the overall simulation becomes more important for larger bottom topographies. This is an interesting result, because Turner and Bridges (2015) showed that the size of $Q(t)-h_{0}$ was directly proportional to the size of the sloshing mode amplitude, but here we observe that despite the wave elevation at $x=0$ and the size of the nonlinearity both decreasing (we know this because the difference between the linear and nonlinear regime results in figure 15 reduces) for increasing $b_{a} / h_{0},\left|Q(t)-h_{0}\right|$ still increases. This suggests a subtle interplay between the topography magnitude and the amplitude of the sloshing modes on the size of the conformal modulus oscillations.

The interior velocity plots in figure 16 show that, unsurprizingly, the fluid at the highest part of the step has an approximate 'depth-averaged' appearance (see panel (a)), with vertical contours, while further down the step the contours look more like those for the deeper water plots in figure 8. This shows that this bed topography gives the flow features of both the deeper water and shallower water results from figures 8 and 11 in the same simulation. In figure 16(c) we see that there are two distinct parts of the free-surface moving in opposite directions (similar to the shallower depth result in figure 11(c)), but like the deeper water results in figure 8 , it is confined to a region close to the free-surface.

For a shallower fluid case, in the transition range between shallow to intermediate depth fluids, with $h_{0}=0.05 \mathrm{~L}$ in figure 17 , we were not able to compute results for the larger values of $b_{a} / h_{0}$, because we find that many more iterations are needed at each time step in order to calculate the mapping $\mathbf{U}_{q}^{-1}$ and hence the simulation run time increased to impractical levels. However, the results presented here do show the same qualitative effect as for the deeper fluid case, but the reduction in the wave amplitude at $x=0$ is less, as is the reduction of the nonlinear fluid effects for larger steps. Figure 17(c) also shows the effect on $Q(t)-h_{0}$ is the same as for the deeper fluid results.

The form of the free-surface profiles $Y(X, t)$ for the step topography are not dissimilar in appearance to those for the homogeneous vessel shown earlier. For example, the nonlinear regime profiles have a wave front appearance like those in figure $7(\mathrm{~b})$, and because 

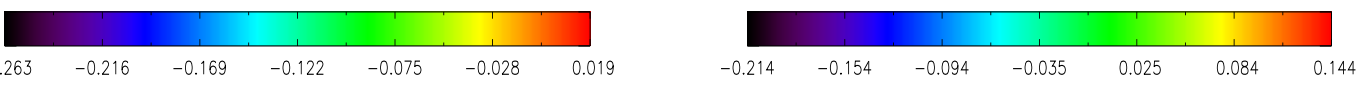

(a)

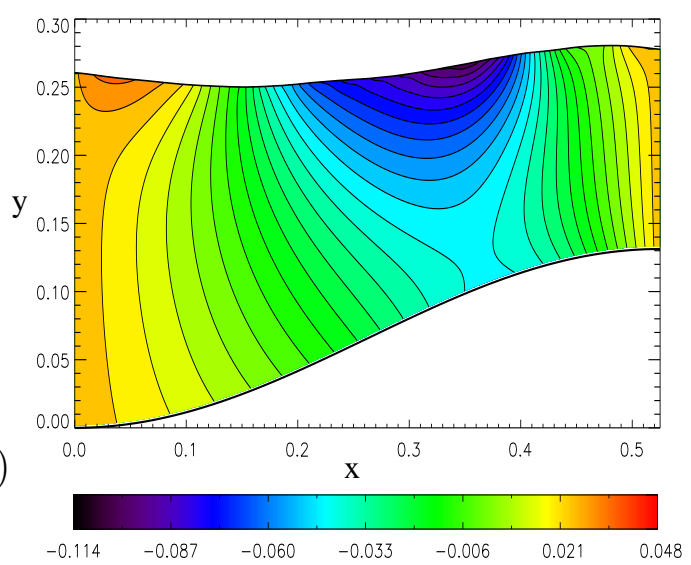

(b)

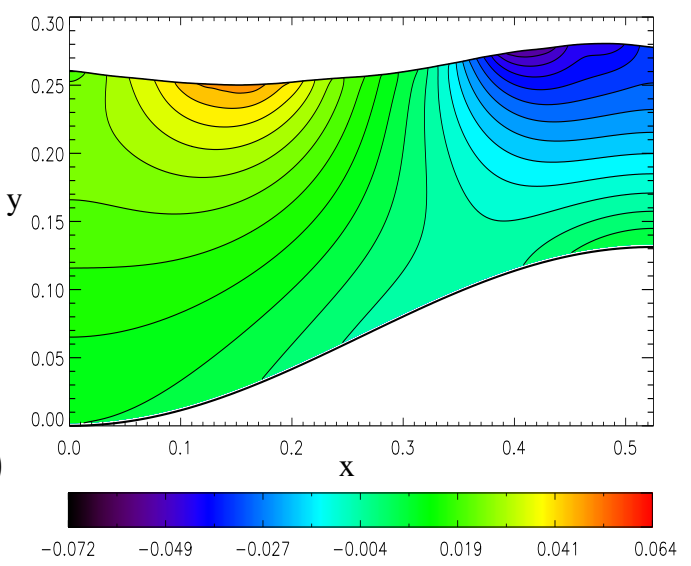

(c)
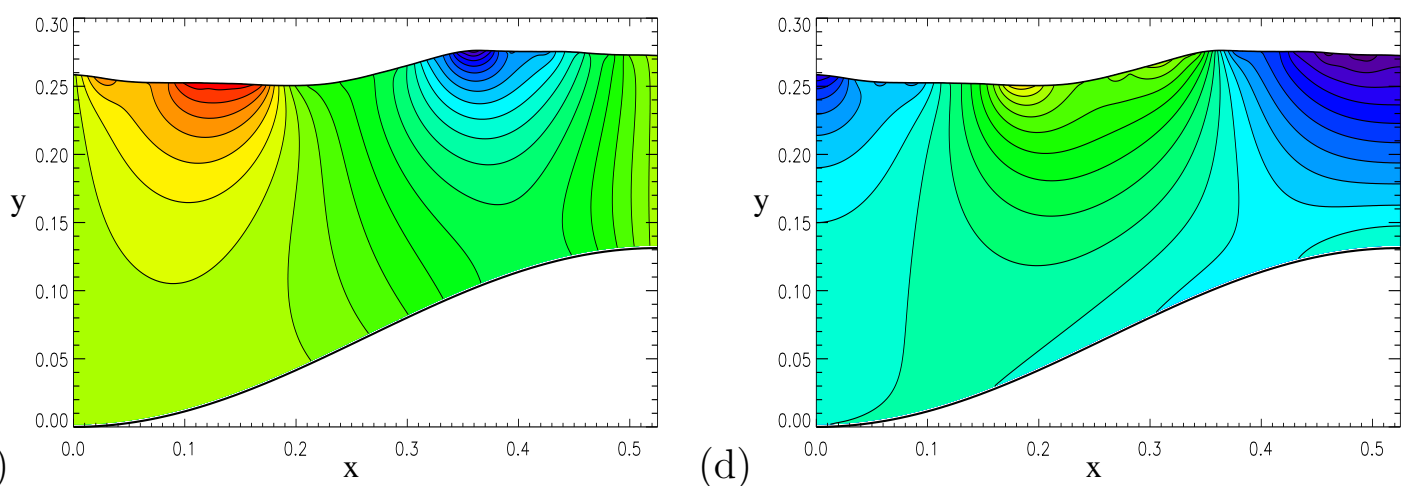

Figure 16: Plot of the contours for (a) $u(x, y)$ and (b) $v(x, y)$ at $t=3.0\left(\omega_{10} t=22.0\right)$ and (c) $u(x, y)$ and $(\mathrm{d}) v(x, y)$ at $t=9.0\left(\omega_{10} t=66.0\right)$ for $h_{0}=0.5 L, b_{a} / h_{0}=0.5$, $\omega_{F} / \omega_{10}=1.3$ and $\epsilon=0.0078$.

of this we do not include these profiles as they do not add anything extra to the analysis.

A potential hazard of inserting a step bottom topography into an existing TLD which is forced at a fixed frequency, is the possibility of exciting a resonance in the system. Figure 18 shows two possible ways in which such a resonance could be excited. In figure 18(a) we consider a forcing frequency $\omega_{F}=0.9 \omega_{10}<\omega_{10}$. Hence as $b_{a}$ is increased the natural frequency of the free-modes decreases (see figure 14) and a resonance occurs with the lowest frequency free-mode for $b_{a} \approx 0.6 h_{0}$. In figure $18(\mathrm{~b})$ we consider the case with $\omega_{F}=1.45 \omega_{10}>\omega_{10}$. Here, for $b_{a} / h_{0}=0.6$ we see large free-surface elevations in comparison to the $b_{a}=0$ case, but this is different from panel (a) because here the resonance occurs with the second free-mode. The significance of this result is that the asymmetric bottom topography breaks the symmetry of the even numbered free-modes, so these modes have to be considered when building in the bottom topography. If the bottom were symmetric then this would not be the case because the initial amplitude of the symmetric modes in the initial condition would be small (or zero) due to the antisymmetric nature of the forcing. In the next section we consider such a symmetric bottom topography. 
(a)

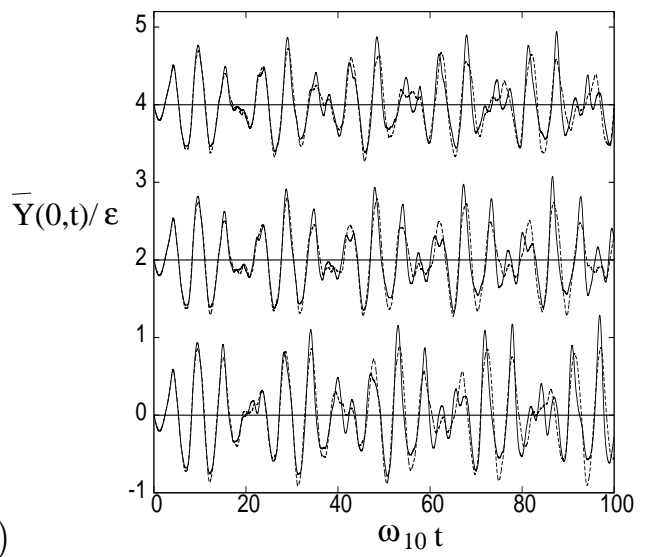

(b)
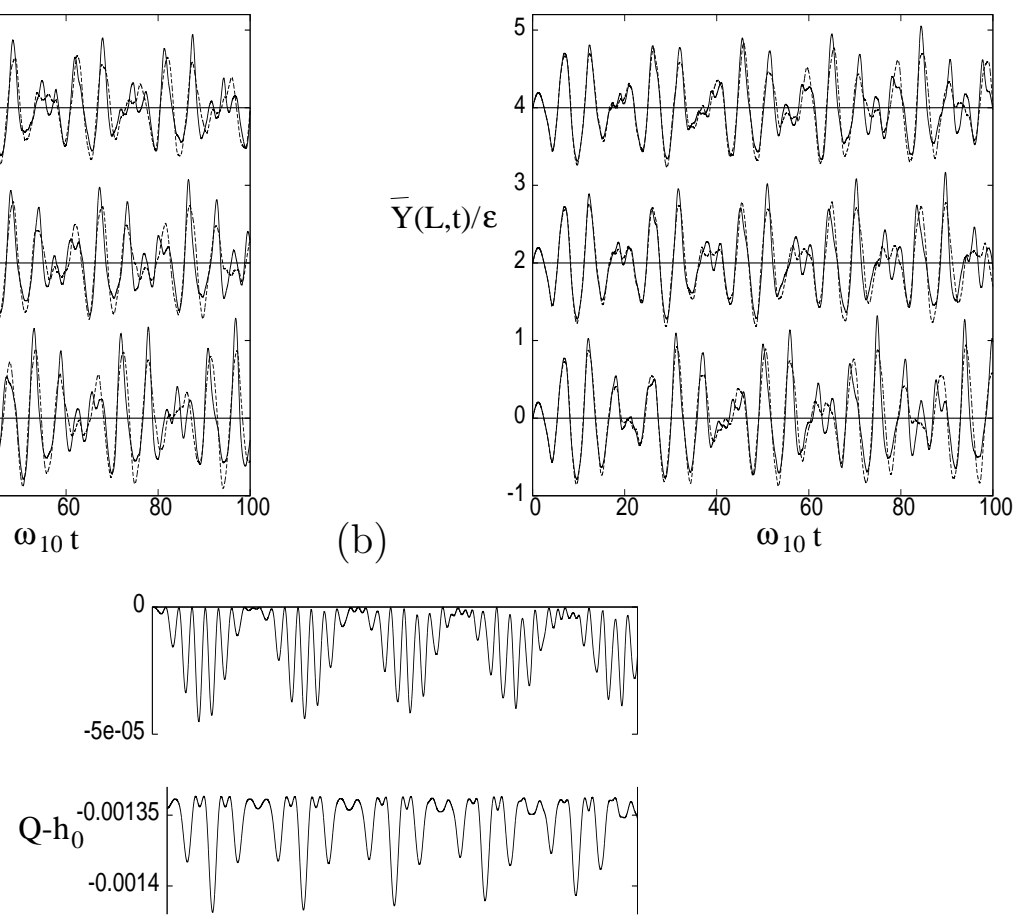

(c)

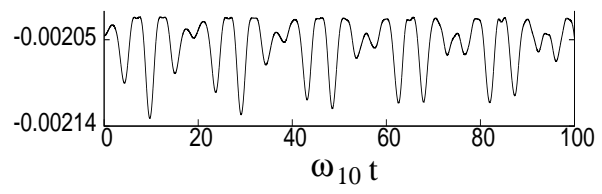

Figure 17: Plot of (a) $\bar{Y}(0, t) / \epsilon$ and (b) $\bar{Y}(L, t) / \epsilon$, when $h_{0}=0.05 L, \omega_{F} / \omega_{10}=1.3$ and $b_{a} / h_{0}=0,0.1$ and 0.15 from bottom to top. In each panel the dashed line corresponds to the linear regime result, $\epsilon=6.3 \times 10^{-5}$, and the solid line is the nonlinear regime result, $\epsilon=0.0032$. Panel (c) plots $Q(t)-h_{0}$ for the nonlinear regime results in panels (a) and (b) with $b_{a} / h_{0}=0,0.1$ and 0.15 from top to bottom. (Note the different $y$-axis scales).

(a)

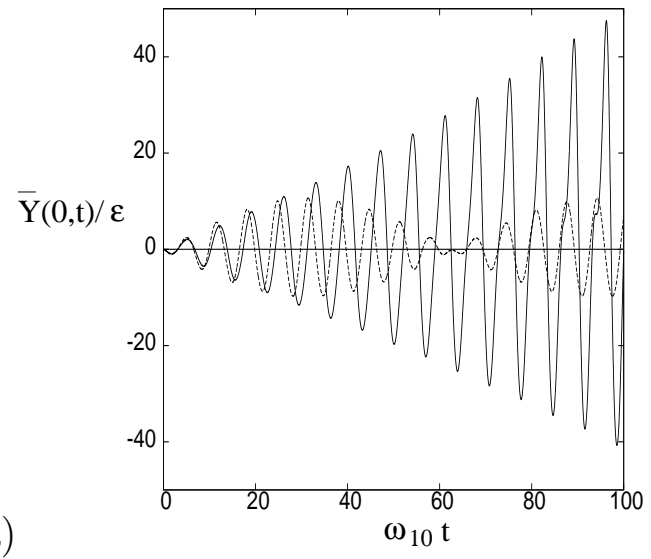

(b)

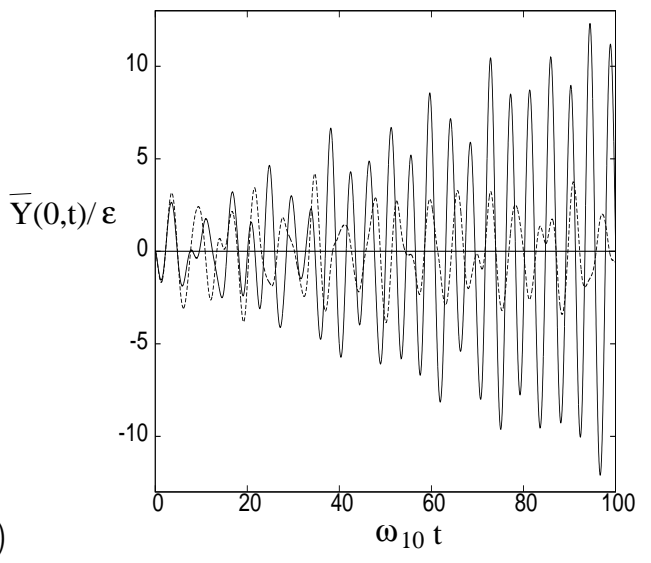

Figure 18: Plot of $\bar{Y}(0, t) / \epsilon$ for $h_{0}=0.5 L$ and $b_{a} / h_{0}=0$ (dashed line) and 0.6 (solid line) with $\epsilon=3.9 \times 10^{-4}$ and (a) $\omega_{F} / \omega_{10}=0.9$ and (b) $\omega_{F} / \omega_{10}=1.45$. 


\subsection{Hump/Trough topography: $b(x)=\frac{1}{2} b_{a}\left(1-\cos \frac{2 \pi x}{L}\right)$}

In this section we consider the symmetric bottom topography given by

$$
b(x)=\frac{1}{2} b_{a}\left(1-\cos \frac{2 \pi x}{L}\right) \quad \text { for } \quad 0 \leq x \leq L .
$$

Because this bottom topography is symmetric, we again have the distinction between symmetric and antisymmetric sloshing modes, with the antisymmetric modes given by the odd values of $n$ and the symmetric modes given by the even values of $n$. Unlike for the topography in $\S 4.2$, here we also consider the case $b_{a}<0$, which corresponds to a trough topography while $b_{a}>0$ corresponds to a hump topography, an example of each is shown by curves 3 and 4 in figure 13 .

(a)

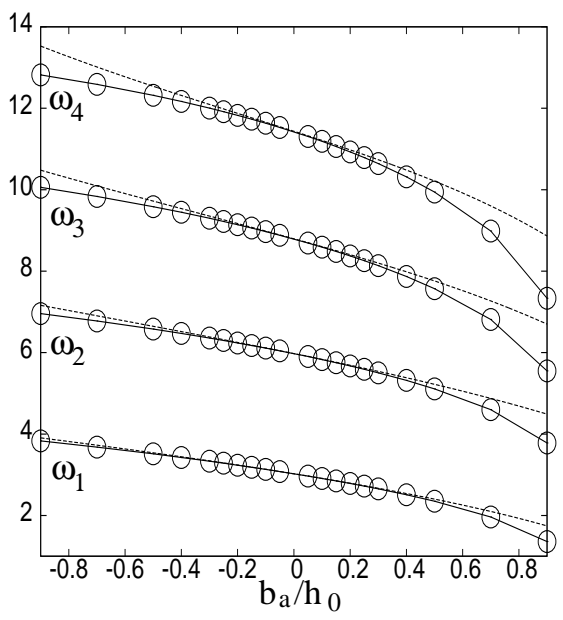

(b)

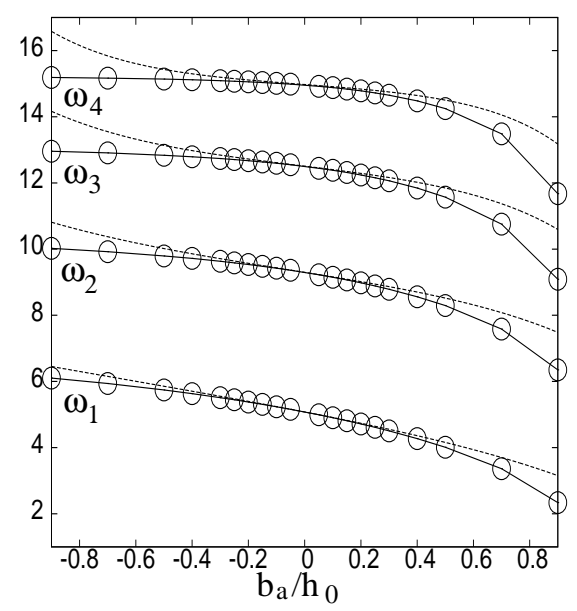

(c)

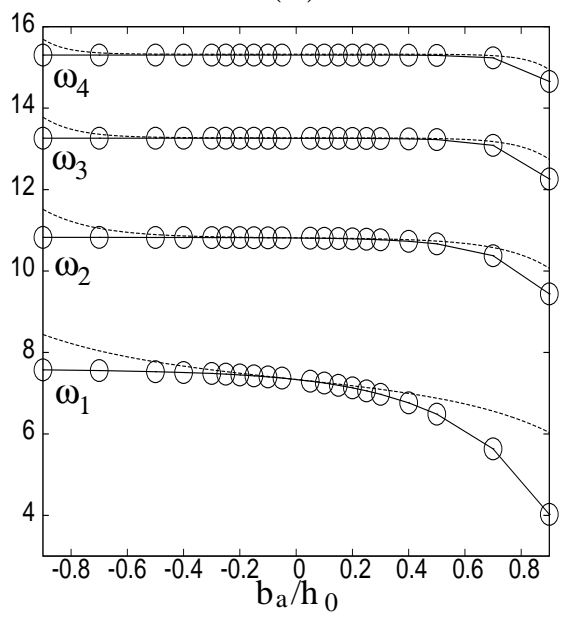

Figure 19: Plot of the natural frequencies $\omega_{n}\left(b_{a} / h_{0}\right)$ for (a) $\delta=0.05$, (b) $\delta=0.15$ and (c) $\delta=0.5$. In each panel the circles represent the results of the numerical simulations and the dashed lines give the asymptotic result (4.49) for the first 4 modes (2 antisymmetric and 2 symmetric).

For this topography the natural frequency of the free-sloshing modes for (a) $\delta=0.05$, (b) $\delta=0.15$ and (c) $\delta=0.5$ are plotted in figure 19. We again observe good agreement with the asymptotic result (4.49) for $\left|b_{a} / h_{0}\right| \lesssim 0.3$, and when $b_{a} / h_{0}>0$ the frequency of 
all the sloshing modes reduces from the $b_{a}=0$ value, while for $b_{a} / h_{0}<0$ the frequency of these modes increases from this value. For $\delta=0.5$ the fundamental mode frequency reduces by $45 \%$ at $b_{a} / h_{0}=0.9$ from the flat bottom result, while for $\delta=0.05$ this reduction is $55 \%$. When $b_{a} / h_{0}=-0.9$ the respective increase of the fundamental mode frequency is $3 \%$ and $26 \%$ for $\delta=0.5$ and 0.05 respectively. We also note that the simulation results always under predict the natural frequency of the free-modes for the larger values of $\left|b_{a} / h_{0}\right|$, as was the case for the step topography in $\S 4.2$.

(a)

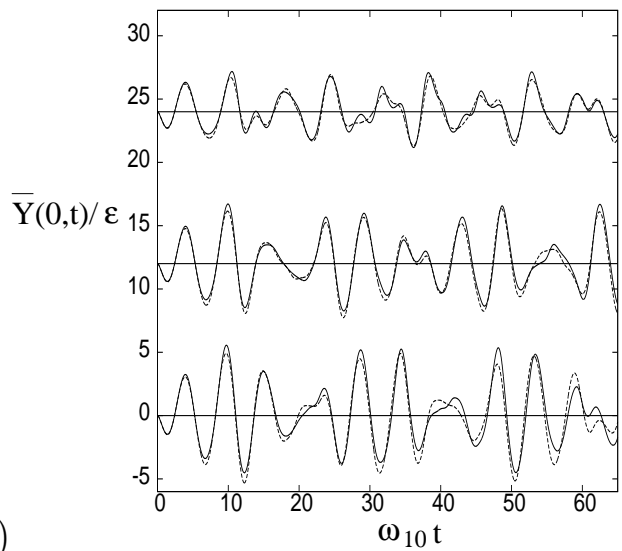

(b)

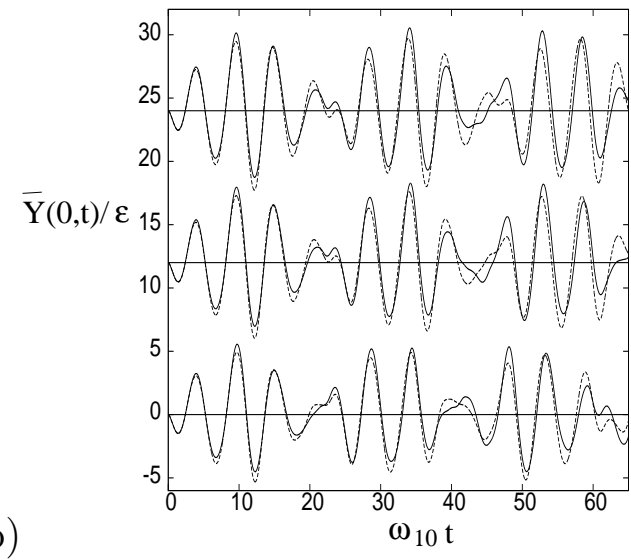

(c)

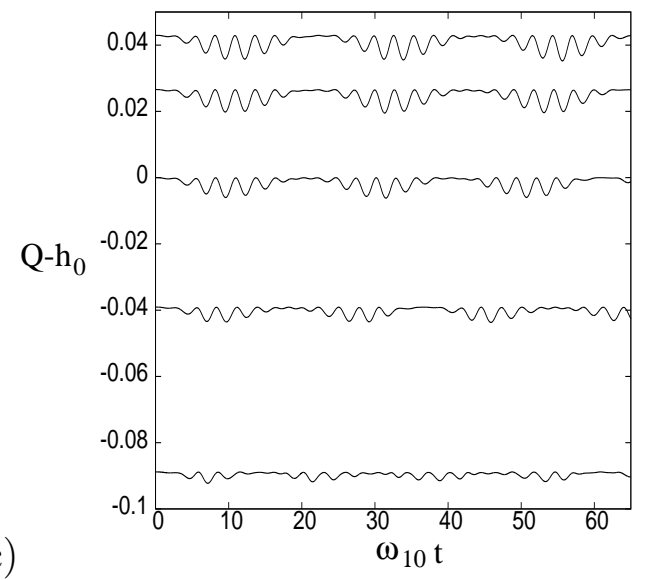

Figure 20: Plot of $\bar{Y}(0, t) / \epsilon$ when $h_{0}=0.5 L, \omega_{F} / \omega_{10}=1.3$ and (a) $b_{a} / h_{0}=0,0.25$ and 0.5 from bottom to top and (b) $b_{a} / h_{0}=0,-0.25$ and -0.5 from bottom to top. In each panel the dashed line corresponds to the linear regime result, $\epsilon=3.0 \times 10^{-4}$ and the solid line is the nonlinear regime result, $\epsilon=0.0078$. Panel (c) plots $Q(t)-h_{0}$ for the nonlinear regime results in panels (a) and (b) with $b_{a} / h_{0}=-0.5,-0.25,0,0.25$ and 0.5 from top to bottom.

In figure 20 we present simulations in both the linear and nonlinear regimes for the case $h_{0}=0.5 L, \omega_{F} / \omega_{10}=1.3$ and $b_{a} / h_{0}=0, \pm 0.25$ and \pm 0.5 . Unlike in $\S 4.2$, the symmetric bottom profile means that we need only consider the free-surface elevation at one end of the vessel. For the case when $b_{a} / h_{0}>0$, i.e. a hump topography, we see that the freesurface elevation is greatly damped, even more so than the antisymmetric bottom in $\S 4.2$, and even when $b_{a} / h_{0}=0.25$ the nonlinear response of the fluid is practically nullified for the chosen forcing magnitude. For the case of a trough topography in panel (b), the free-surface elevation does not change a great deal, and while the nonlinear effects appear 
to be larger for $b_{a} / h_{0}=-0.5$ than for $b_{a} / h_{0}=0$, the difference is not significant. This is unsurprising though, as $h_{0}=0.5 \mathrm{~L}$ is already in the transition region from an intermediate to a deep fluid, so inserting a trough just moves the vessel geometry closer to the deep water $\operatorname{limit}(\tanh (\pi \delta) \approx 1, \delta \approx 0.9)$.

(a)

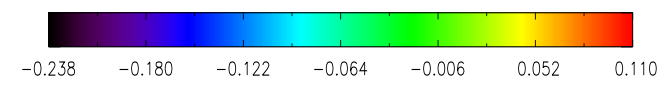

(c)

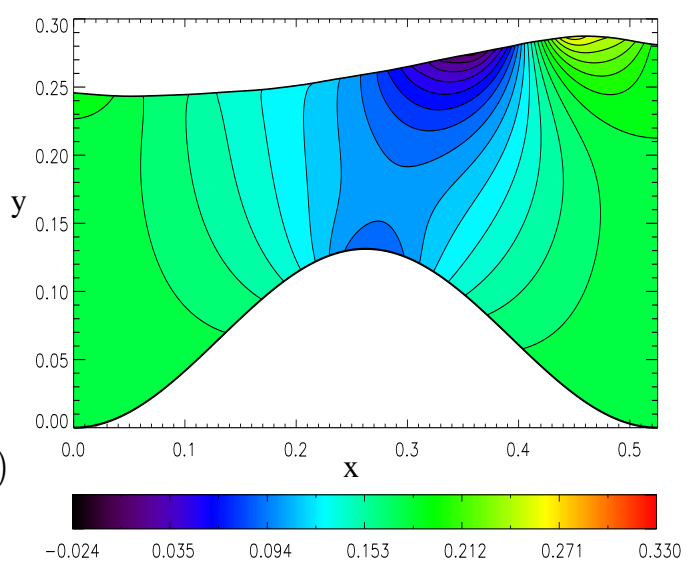

(b)
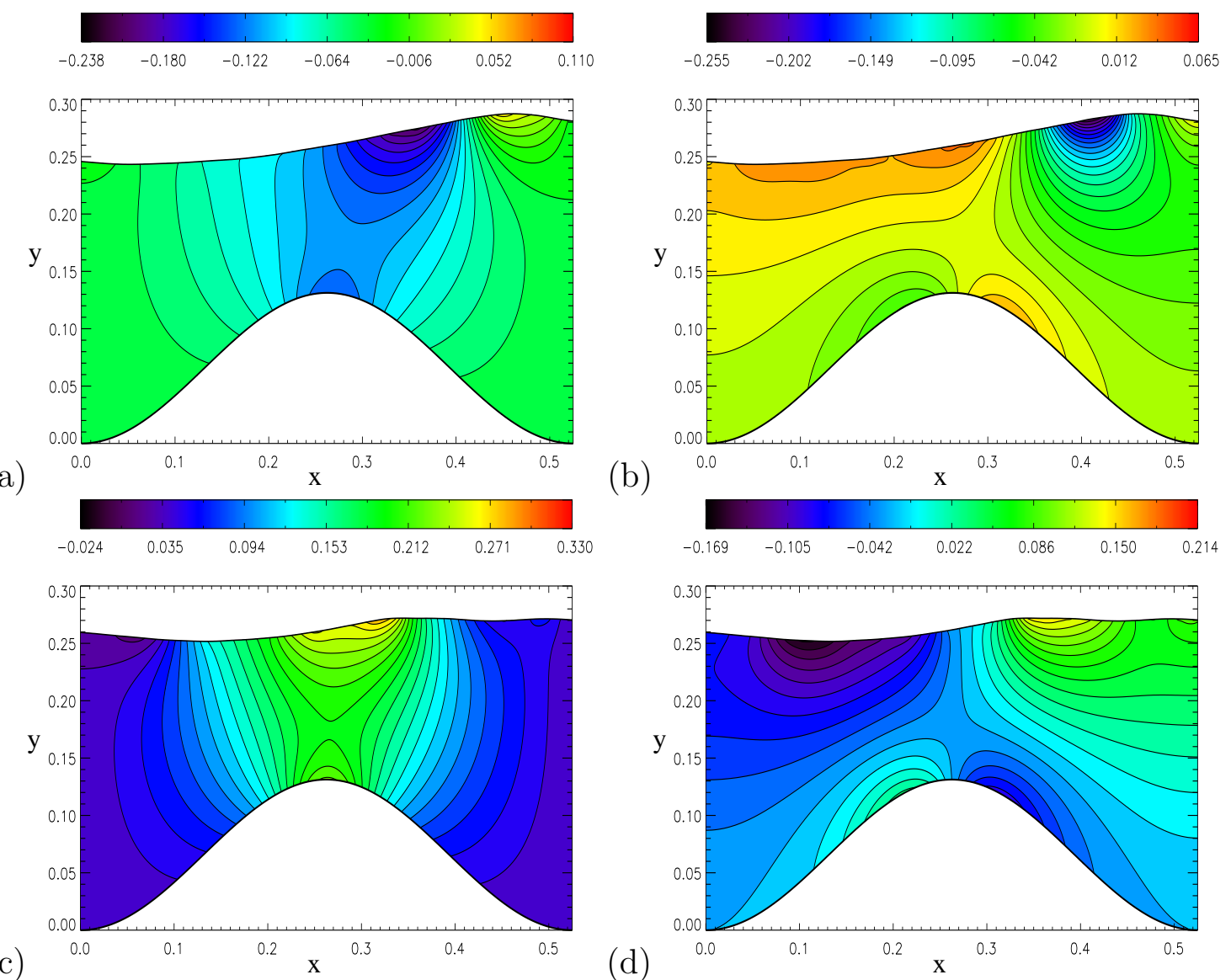

(d)

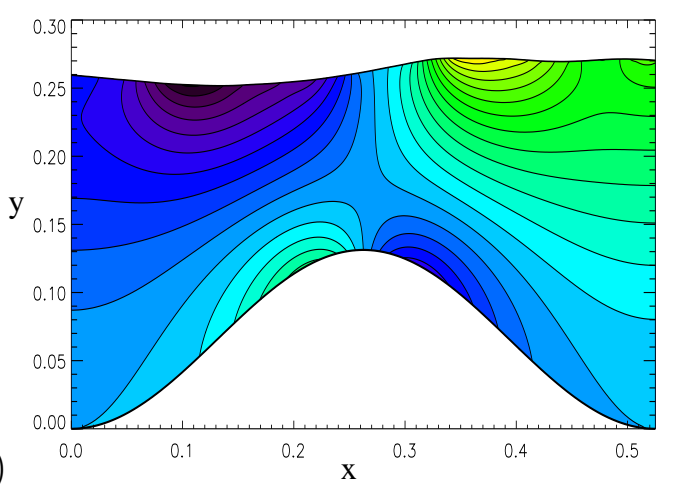

Figure 21: Plot of the contours for (a) $u(x, y)$ and (b) $v(x, y)$ at $t=3.0\left(\omega_{10} t=22.0\right)$ and (c) $u(x, y)$ and (d) $v(x, y)$ at $t=7.5\left(\omega_{10} t=55.0\right)$ for $h_{0}=0.5 L, b_{a} / h_{0}=0.5$, $\omega_{F} / \omega_{10}=1.3$ and $\epsilon=0.0032$.

The interior velocity plots in figure 21 show that, unlike the step case in figure 16, the horizontal velocity plot does not have a depth averaged appearance as it moves over the highest point of the hump. However, we again see that the faster velocities are again confined to a region close to the free-surface.

The main conclusion from this section is these results show that a TLD with a hump topography is the most effective topography when it comes to damping out the fluid sloshing motion and hence reducing the fluid stress on the vessel walls. This is in line with the experimental findings of Idir et al. (2009).

\subsection{Broad and narrow topographies}

In this final section we consider the effect on the fluid motion by considering TLDs with inhomogeneous bottom topographies consisting of broader and narrower steps from $\S 4.2$ 
and broader and narrower humps from $§ 4.3$. In order to achieve this we introduce the bottom topography function

$$
b(x)=\left\{\begin{array}{ll}
b_{a}\left(1-\mathrm{cn}^{2}\left(n K(m) \frac{x}{L}, m\right)\right) & m \geq 0 \\
b_{a} \operatorname{cn}^{2}\left(n K(|m|) \frac{x}{L},|m|\right) & m<0
\end{array} \quad \text { for } 0 \leq x \leq L,\right.
$$

where

$$
K(m)=\int_{0}^{\pi / 2} \frac{d \theta}{\left(1-m^{2} \sin ^{2} \theta\right)^{1 / 2}}
$$

and

$$
\operatorname{cn}(u, m)=\cos (\phi) \quad \text { with } \quad u=\int_{0}^{\phi} \frac{d \theta}{\left(1-m^{2} \sin ^{2} \theta\right)^{1 / 2}} .
$$

Here $\operatorname{cn}(u, m)$ is the Jacobian elliptic cosine function (Bowman, 1953) and $n=1$ for the step profile and $n=2$ for the hump profile. The parameter $m$ defines the broadness of the function $b(x)$, and when $m=0$ the topographies are the same as those in $\S 4.2$ and $\S 4.3$ for $n=1$ and $n=2$ respectively. For $|m| \lesssim 0.5$ the deviation of $b(x)$ from these previous profiles is small, and in the limits $m \rightarrow 1, b(x) \rightarrow b_{a}=$ constant, while as $m \rightarrow-1 b(x) \rightarrow 0$, except at $x=L / n$. A plot of $b(x)$ for various values of $m$ are given in figure 22 , along with the profiles of $d b / d x$ which are given by

(a)
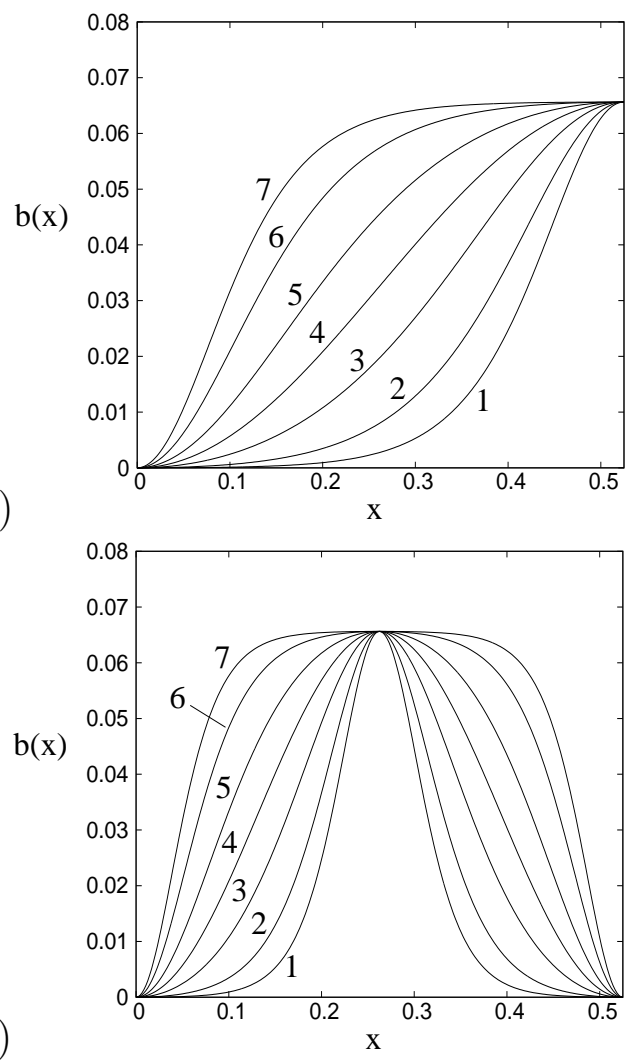

(b)

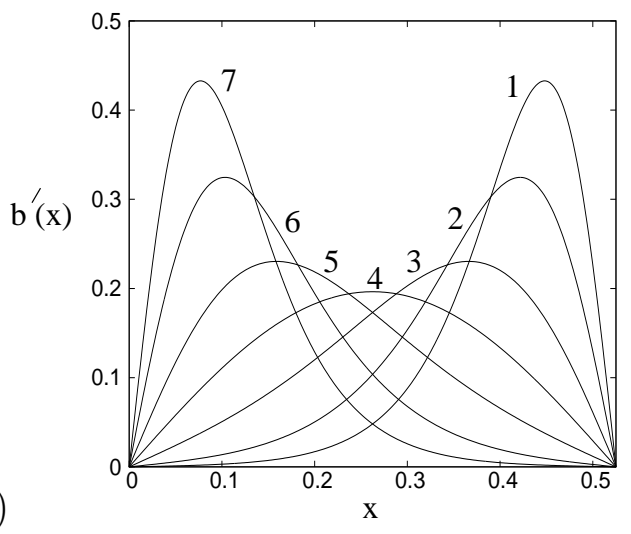

(d)

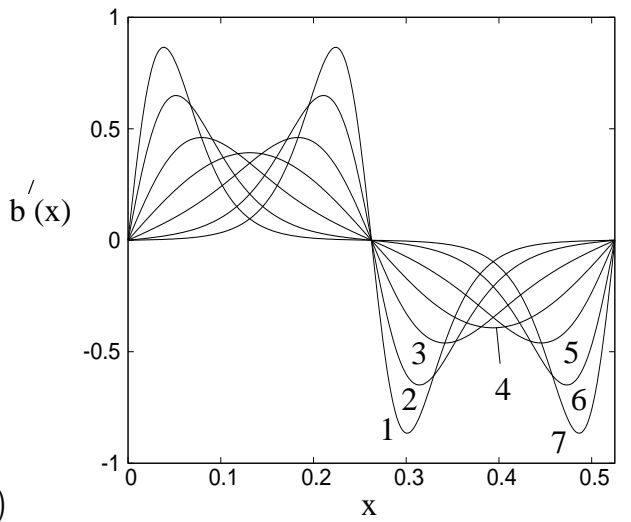

Figure 22: Plot of $b(x)$ and $b^{\prime}(x)$ given in (4.50) and (4.51) for (a,b) $n=1$ and (c,d) $n=2$ with $b_{a}=0.25 h_{0}$ and $\delta=0.5$. In each panel the profiles $m=-0.999,-0.99,-$ $0.9,0,0.9,0.99,0.999$ are numbered $1-7$ respectively. 


$$
\frac{d b}{d x}=\operatorname{sgn}(m) \frac{2 n b_{a} K(|m|)}{L}\left[1-m^{2} \operatorname{sn}^{2}\left(n K(|m|) \frac{x}{L},|m|\right)\right]^{1 / 2} \operatorname{cn}\left(n K(|m|) \frac{x}{L},|m|\right) \operatorname{sn}\left(n K(|m|) \frac{x}{L},|m|\right)
$$

where

$$
\operatorname{sn}(u, m)=\sin (\phi),
$$

is the Jacobian elliptic sine function (Bowman, 1953) and $\operatorname{sgn}(m)$ is the sign function. The elliptic sine function is related to the elliptic cosine function via $\operatorname{sn}^{2}(u, m)=1-\operatorname{cn}^{2}(u, m)$.

For the step profile $(n=1)$, figure 22(a) shows that the step is narrow and concentrated closer to $x=L$ for large negative $m$, while for large positive $m$ the step is broad, with a small cavity region close to $x=0$. Figure 22 (b) also shows that as $|m|$ increases the gradient of the step increases, with the lowest gradient occurring for $m=0$.

(a)

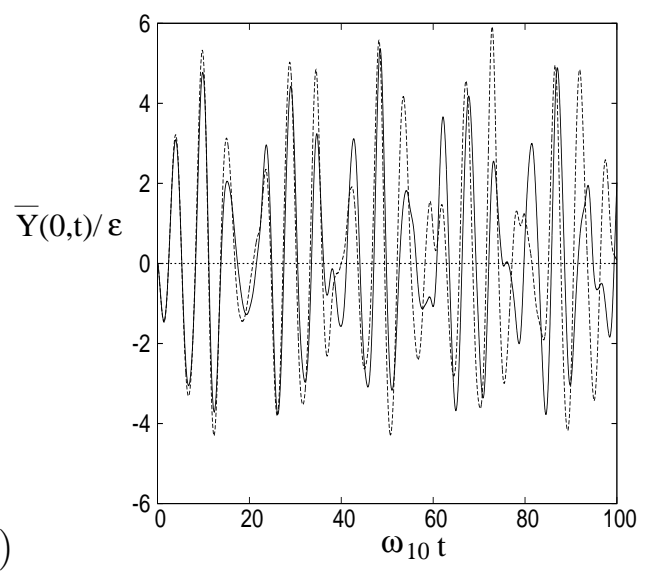

(c)

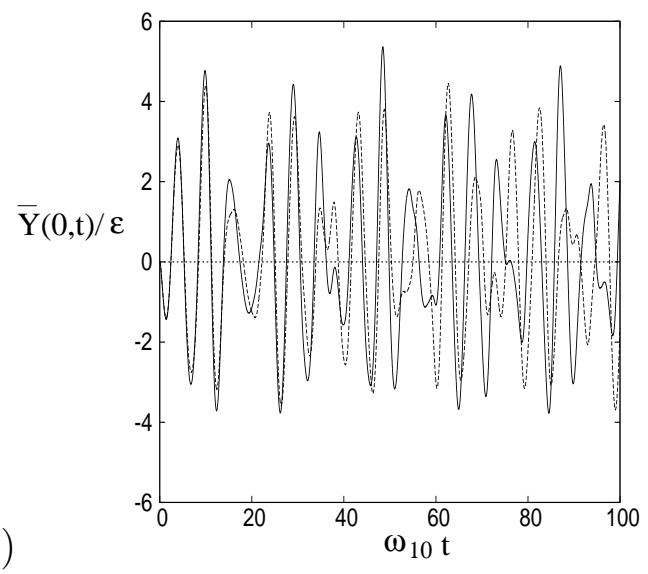

(b)

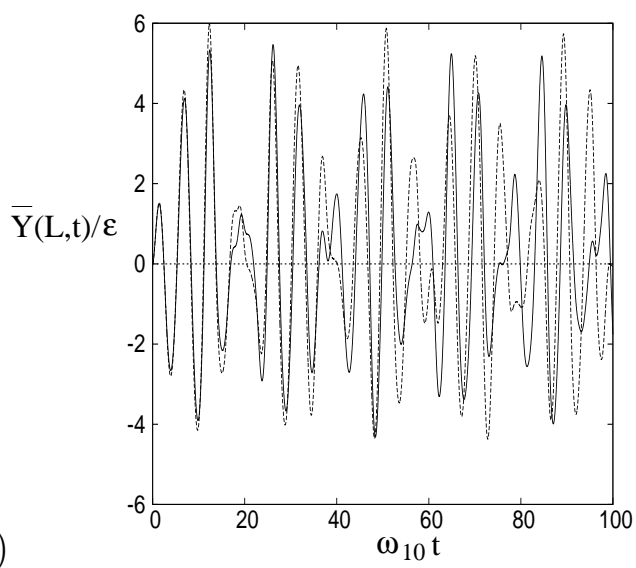

(d)

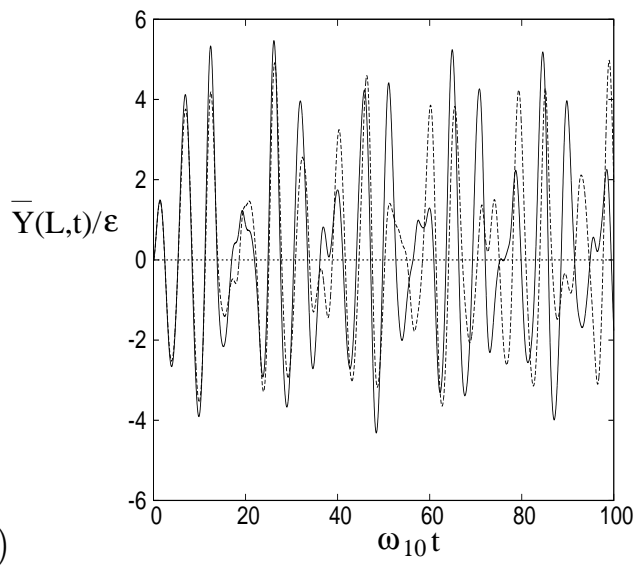

Figure 23: Plot of $\bar{Y}(0, t) / \epsilon$ and $\bar{Y}(L, t) / \epsilon$, when $h_{0}=0.5 L, \omega_{F} / \omega_{10}=1.3, b_{a} / h_{0}=0.25$ for the profiles in (4.50) with $n=1$ and (a,b) $m=0,-0.999$, and (c,d) $m=0,0.999$ given by the solid, dashed and dotted lines respectively. The magnitude of the forcing is $\epsilon=0.0078$, hence these are nonlinear regime simulations.

In figure 23 we focus on simulations for the nonlinear regime sloshing amplitude $\epsilon=$ 0.0078 with $b_{a} / h_{0}=0.25$ and $\delta=0.5$, and investigate the effect on the free-surface elevation of broadening/narrowing the step topography. These results appear to show no real long time trend, because the results diverge from one another as time increases, but a careful inspection of the peak and trough amplitudes shows that in general the narrower step leads to larger amplitude free-surface oscillations than for the broader step, at least 
over the time scale investigated here. When we compare the conformal modulus evolution

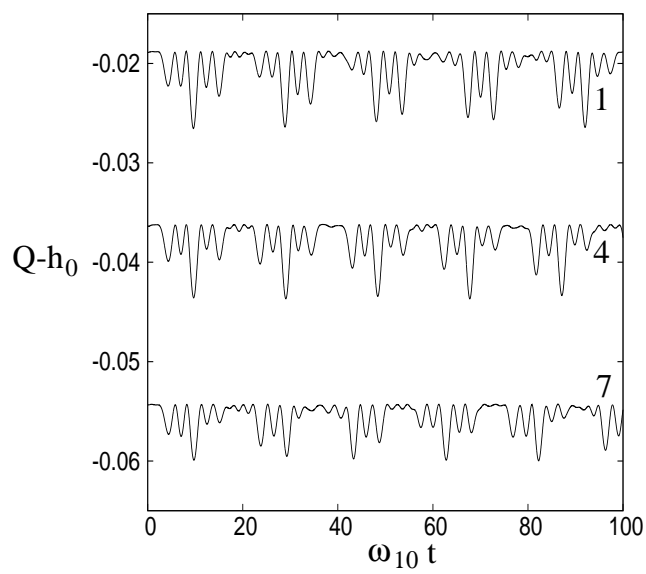

Figure 24: Plot of $Q-h_{0}$ for the results in figure 23. Here the results are numbered with the same convention as those in figure $22(\mathrm{a})$.

in figure 24 for these simulations we see that as the step broadens (result 1 through to 7 ) the average value of $Q(t)$ reduces and the magnitude of the oscillations from the mean value reduces slightly too. This is not surprising though, because for the narrower step there is effectively a deeper expanse of fluid in the vessel than for the broader step, and we saw in $\S 4.2$ that for shallower fluids the nonlinear effect in the fluid reduces, and the maximum magnitude of $\bar{Y}(0, t) / \epsilon$ also reduces.

For the hump profile $(n=2)$, figure 22(c) shows that the step is narrow for large negative values of $m$, while for large positive $m$ the step is broad. Figure 22(b) also shows that as $|m|$ increases the gradient either side of the hump increases, with the lowest gradient occurring for $m=0$.

(a)

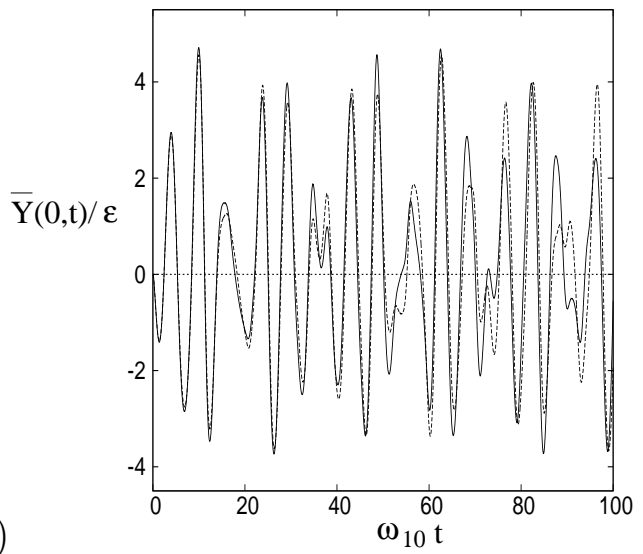

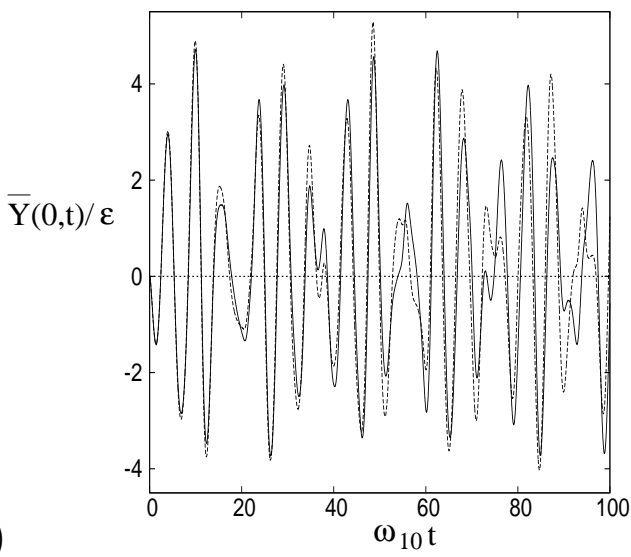

Figure 25: Plot of $\bar{Y}(0, t) / \epsilon$, when $h_{0}=0.5 L, \omega_{F} / \omega_{10}=1.3, b_{a} / h_{0}=0.25$ for the profiles in (4.50) with $n=2$ and (a) $m=0,-0.99$, and (b) $m=0,0.99$ given by the solid, dashed and dotted lines respectively. The magnitude of the forcing is $\epsilon=0.0078$, hence these are nonlinear regime simulations.

The nonlinear regime free-surface elevation profiles in figure 25 for the broadening 
humps behave in a similar manner as for the steps in figure 23. Again the results for different values of $m$ diverge from one another as time increases, however, this time the narrower steps (panel (a)) generally reduce the free-surface amplitude at $x=0$, while for the broader steps the free-surface amplitude is slightly increased. Note that the amount by which $\bar{Y}(0, t) / \epsilon$ is increased or reduced in this case is much less than for the step profiles.

Thus we can conclude that the overall width of the step/hump is not greatly significant in reducing the nonlinear feedback of the fluid, its mere presence and hence its magnitude makes the largest effect. The results in figure 25 suggest that the most effective damping topography for the nonlinear fluid response is likely to be an infinitely thin hump, or equivalently, a submerged baffle such as that in Evans and McIver (1987).

\section{Conclusions and discussion}

This paper examined the effect of an inhomogeneous bottom topography on the free surface elevation of a sloshing fluid in a periodic, horizontally forced vessel, such as a Tuned Liquid Damper (TLD). Results were simulated using a time-dependent conformal mapping technique to map the complex geometry, including the position of the unknown free-surface, to a rectangle in the computational domain with a time-dependent conformal modulus. As the mapping was time-dependent the conformal modulus had to be found as part of the solution making the numerical method computationally complex. The main advantage of this scheme over other potential flow schemes, such as that of Frandsen (2004) for example, is the scheme is constructed such that only the time integration of the two free-surface boundary conditions is required. This made it computationally fast, allowed for high spatial resolution as only a 1-dimensional grid was required, and bottom topography effects can be easily incorporated. The scheme detailed in this paper is robust and results were presented for invsicid, non-breaking sloshing waves in a flat bottomed vessel for mean fluid depths from the intermediate depth/deep water border, down to the intermediate depth/shallow water border. Resonant sloshing simulations were also presented and showed that the scheme can simulate large amplitude nonlinear waves up to approximately 60 times the forcing amplitude.

Simulations were also presented for an asymmetric 'step' profile as well as a symmetric 'hump' profile. In both cases, analysis of the free-sloshing modes found that their natural frequency decreased as the ratio of the bottom topography magnitude to the average fluid depth increased. Nonlinear regime simulations showed that the hump topography was the most effective profile for reducing the nonlinear response of the fluid, as well as for reducing the overall amplitude of the free-surface elevations. This result is perhaps not a surprize, as previous studies have demonstrated the effectiveness of baffles (limit of an infinitely thin hump) in damping hydrodynamic effects in rectangular tanks (Biswal et al., 2006; Younes et al., 2007; Akyildiz, 2012; Xue et al., 2012). However, as far as the author is aware, this was the first systematic study of bottom topography in rectangular vessels leading to this conclusion. This suppression of free-surface waves is significant because smaller free-surface elevations at the side walls mean smaller stresses on the vessel, and hence are less likely to lead to damage, in the case of a prescribed horizontal forcing. This suppression of the free-surface waves is similar to that already seen in TLDs in the works of Tait and co-workers who use surface piercing damping screens (essentially surface 
piercing porous baffles) to damp the free-surface waves (Tait et al., 2005; Cassolato et al., 2011).

It was also highlighted in this study that adding inhomogeneous bottoms into existing vessels, such as TLDs or fuel tanks could be problematic because one could excite a resonance frequency in the system which was not present in the homogeneous vessel. This is more likely for a step profile because the asymmetric topography breaks the distinction between symmetric and antisymmetric sloshing modes, giving the possibility of a resonance with more free modes. Such a resonance would again lead to large fluid responses and large stresses on the vessel walls.

One scenario not considered in this paper is, what happens if the forcing is turned off after some time, and the vessel is free to move under the motion of the fluid restricted by a spring as in the case of a TLD, or with planer translations and roll, as in the case of a ship for example? In these cases the sloshing fluid produces a force on the vessel walls, causing it to move which hence produces a subsequent force on the fluid. This dynamic coupling effect has been widely studied in homogeneous bottomed vessels (Cooker, 1994; Yu, 2010; Alemi Ardakani and Bridges, 2010; Herczyński and Weidman, 2012; Alemi Ardakani et al., 2012; Turner et al., 2015a) and interesting phenomena such as energy transfer between the sloshing modes has been identified (Turner and Bridges, 2013). The results of this paper suggest that the inclusion of a step or hump topography would decrease the feedback mechanism from the fluid to the vessel as these topographies reduce the size of the fluid amplitudes at the side walls. One would expect this to stabilize the motion of the vessel in the dynamically coupled system. However, the literature on this phenomenon for the dynamically coupled system is small, and the confirmation of this expectation is left as an ongoing study.

\section{Acknowledgments}

This work is supported by the EPSRC under grant number EP/K008188/1. Due to confidentiality agreements with research collaborators, supporting data can only be made available to bona fide researchers subject to a non-disclosure agreement. Details of the data and how to request access are available from the University of Surrey publications repository: researchdata@surrey.ac.uk

\section{References}

Abramson, H. (1966). The Dynamic Behavior of Liquids in Moving Containers. NASA SP-106 (Washington D. C.).

Akyildiz, H. (2012). A numerical study of the effects of the vertical baffle on liquid sloshing in two-dimensional rectangular tank. J. Sound Vibr., 331(1), 41-52.

Akyildiz, H. and Ünal, E. N. (2005). Experimental investigation of pressure distribution on a rectangular tank due to the liquid sloshing. Ocean Engineering, 32(11), 1503-1516.

Akyildız, H. and Ünal, E. N. (2006). Sloshing in a three-dimensional rectangular tank: numerical simulation and experimental validation. Ocean Engineering, 33(16), 21352149 . 
Alemi Ardakani, H. and Bridges, T. J. (2010). Dynamic coupling between shallow-water sloshing and horizontal vehicle motion. Europ. J. Appl. Math, 21, 479-517.

Alemi Ardakani, H., Bridges, T. J., and Turner, M. R. (2012). Resonance in a model for Cooker's sloshing experiment. Euro. J. Mech. B/Fluids, 36, 25-38.

Armenio, V. and La Rocca, M. (1996). On the analysis of sloshing of water in rectangular containers: numerical study and experimental validation. Ocean Engineering, 23(8), 705-739.

Baker, G. R., Meiron, D. I., and Orszag, S. A. (1989). Generalized vortex methods for free surface flow problems. ii: Radiating waves. J. Sci. Comput., 4(3), 237-259.

Biswal, K. C., Bhattacharyya, S. K., and Sinha, P. K. (2006). Non-linear sloshing in partially filled containers with baffles. Int. J. Numer. Methods Eng., 68, 317-337.

Bowman, F. (1953). Introduction to Elliptic Functions. English Universities Press.

Bridges, T. J. and Donaldson, N. M. (2011). Variational principles for water waves from the viewpoint of a time dependent moving mesh. Mathematika, 57, 147-173.

Broyden, C. G. (1965). A class of Methods for Solving Nonlinear Simultaneous Equations. Mathematics of Computation, 19(92), 577-593.

Cassolato, M. R., Love, J. S., and Tait, M. J. (2011). Modelling of a tuned liquid damper with inclined damping screens. Struct. Control Health Monit., 18(6), 674-681.

Choi, W. and Camassa, R. (1999). Exact evolution equations for surface waves. J. Eng, Mech., 125, 756-760.

Choun, Y.-S. and Yun, C.-B. (1996). Sloshing characteristics in rectangular tanks with a submerged block. Computers Structs., 61(3), 401-413.

Cooker, M. J. (1994). Water waves in a suspended container. Wave Motion, 20, 385-395.

Dyachenko, A. I., Kuznetsov, E. A., Spector, M. D., and Zakharov, V. E. (1996). Analytical description of the free surface dynamics of an ideal fluid (canonical formalism and conformal mapping). Phys. Lett. A, 221, 73-79.

Dyachenko, A. I., Zakharov, V. E., and Kuznetsov, E. A. (1999). Nonlinear dynamics of the free surface of an ideal fluid. Plasma Phys. Rep., 22, 916-928.

Dyachenko, A. I., Korotkevich, A. O., and Zakharov, V. E. (2004). Weak turbulent kolmogorov spectrum for surface gravity waves. Phys. Rev. Lett., 92(13), 134501.

Evans, D. and McIver, P. (1987). Resonant frequencies in a container with a vertical baffle. Journal of Fluid Mechanics, 175, 295-307.

Faltinsen, O. M. and Timokha, A. N. (2009). Sloshing. Cambridge University Press (Cambridge). 
Firouz-Abadi, R. D., Haddadpour, H., Noorian, M. A., and Ghasemi, M. (2008). A 3d bem model for liquid sloshing in baffled tanks. Int. J. Numer. Meth. Eng., 76(9), 1419-1433.

Frandsen, J. B. (2004). Sloshing motions in excited tanks. J. Comput. Phys., 196, 53-87.

Gardarsson, S., Yeh, H., and Reed, D. (2001). Behavior of sloped-bottom tuned liquid dampers. Journal of engineering mechanics, 127(3), 266-271.

Gerrits, J. (2001). Dynamics of liquid-filled spacecraft. PhD Thesis, Rijks University Groningen, Holland.

Grilli, S. T. (1998). Depth inversion in shallow water based on nonlinear properties of shoaling periodic waves. Coastal Engineering, 35(3), 185-209.

Grilli, S. T. and Watts, P. (1999). Modeling of waves generated by a moving submerged body. applications to underwater landslides. Engineering Analysis with boundary elements, 23(8), 645-656.

Grilli, S. T., Subramanya, R., Svendsen, I. A., and Veeramony, J. (1994). Shoaling of solitary waves on plane beaches. Journal of Waterway, Port, Coastal, and Ocean Engineering, 120(6), 609-628.

Grilli, S. T., Svendsen, I. A., and Subramanya, R. (1997). Breaking criterion and characteristics for solitary waves on slopes. Journal of waterway, Port, Coastal, and Ocean Engineering, 123(3), 102-112.

Herczyński, A. and Weidman, P. D. (2012). Experiments on the periodic oscillation of free containers driven by liquid sloshing. J. Fluid Mech., 693, 216-242.

Ibrahim, R. A. (2005). Liquid Sloshing Dynamics. Cambridge University Press (Cambridge).

Idir, M., Ding, X., Lou, M., and Chen, G. (2009). Fundamental frequency of water sloshing waves in a sloped-bottom tank as tuned liquid damper. In Structures Congress 2009: Dont Mess with Structural Engineers: Expanding Our Role, pages 1-10. ASCE.

Kareem, A., Kijewski, T., and Tamura, Y. (1999). Mitigation of motions of tall buildings with specific examples of recent applications. Wind and structures, 2(3), 201-251.

Moiseyev, N. N. and Rumyantsev, V. V. (1968). Dynamic Stability of Bodies Containing Fluid. Springer-Verlag (New York).

Papamichael, N. and Stylianopoulos, N. (2010). Numerical conformal mapping: Domain decomposition and the mapping of quadrilaterals. World Scientific (Singapore).

Phillips, N. A. (1957). A coordinate system having some special advantages for numerical forecasting. Journal of Meteorology, 14(2), 184-185.

Putnam, J. A. and Johson, J. W. (1949). The dissipation of wave energy by bottom friction. Eos, Transactions American Geophysical Union, 30(1), 67-74. 
Ruban, V. (2004). Water waves over a strongly undulating bottom. Physical Review E, $\mathbf{7 0}(6), 066302$.

Ruban, V. P. (2005). Water waves over a time-dependent bottom: Exact description for 2d potential flows. Physics Letters A, 340(1), 194-200.

Tait, M. J., El Damatty, A. A., Isyumov, N., and Siddique, M. R. (2005). Numerical flow models to simulate tuned liquid dampers (tld) with slat screens. J. Fluid Struct., 20(8), 1007-1023.

Tuck, E. O. (1974). The effect of a surface layer of viscous fluid on the wave resistance of a thin ship. J. Ship Research, 18(4).

Turner, M. R. and Bridges, T. J. (2013). Nonlinear energy transfer between fluid sloshing and vessel dynamics. J. Fluid Mech., 719, 606-636.

Turner, M. R. and Bridges, T. J. (2015). Time-dependent conformal mapping of doublyconnected regions. Adv. Comp. Math (submitted).

Turner, M. R., Alemi Ardakani, H., and Bridges, T. J. (2015a). Instability of sloshing motion in a vessel undergoing pivoted oscillations. J. Fluid Struct., 52, 166-180.

Turner, M. R., Bridges, T. J., and Alemi Ardakani, H. (2015b). The pendulum-slosh problem: Simulation using a time-dependent conformal mapping. J. Fluid Struct., 59, 202-223.

Viotti, C., Dutykh, D., and Dias, F. (2013). The conformal-mapping method for surface gravity waves in the presence of variable bathymetry and mean current. Procedia IUTAM, pages $1-13$.

Wang, J. and Joseph, D. D. (2006). Purely irrotational theories of the effect of the viscosity on the decay of free gravity waves. J. Fluid Mech., 559, 461-472.

Wilkening, J. and Yu, J. (2012). Overdetermined shooting methods for computing standing water waves with spectral accuracy. Comp. Science and Discovery, 5, 014017.

Xue, M.-A., Zheng, J., and Lin, P. (2012). Numerical simulation of sloshing phenomena in cubic tank with multiple baffles. Journal of Applied Mathematics, 2012.

Younes, M. F., Younes, Y. K., El-Madah, M., Ibrahim, I. M., and El-Dannanh, E. H. (2007). An experimental investigation of hydrodynamic damping due to vertical baffle arrangements in a rectangular tank. Proc. IMeche. Part M: J. Eng. Maritime Environ, 221(3), 115-123.

Yu, J. (2010). Effects of finite water depth on natural frequencies of suspended water tanks. Stud. Appl. Math., 125, 337-391. 